\title{
Rare top-quark decays to Higgs boson in MSSM
}

\author{
A. Dedes, ${ }^{a, b}$ M. Paraskevas, ${ }^{a}$ J. Rosiek, ${ }^{c}$ K. Suxho ${ }^{a}$ and K. Tamvakis ${ }^{a}$ \\ ${ }^{a}$ Department of Physics, Division of Theoretical Physics, \\ University of Ioannina, GR 45110, Greece \\ ${ }^{b}$ University of Athens, Physics Department, \\ Nuclear and Particle Physics section, GR 15771 Athens, Greece \\ ${ }^{c}$ Institute of Theoretical Physics, Warsaw University, \\ Hoza 69, 00-681 Warsaw, Poland \\ E-mail: adedes@cc.uoi.gr, mparask@grads.uoi.gr, \\ janusz.rosiek@fuw.edu.pl, csoutzio@cc.uoi.gr, tamvakis@uoi.gr
}

ABSTRACT: In full one-loop generality and in next-to-leading order in QCD, we study rare top to Higgs boson flavour changing decay processes $t \rightarrow q h$ with $q=u, c$ quarks, in the general MSSM with R-parity conservation. Our primary goal is to search for enhanced effects on $\mathcal{B}(t \rightarrow q h)$ that could be visible at current and high luminosity LHC running. To this end, we perform an analytical expansion of the amplitude in terms of flavour changing squark mass insertions that treats both cases of hierarchical and degenerate squark masses in a unified way. We identify two enhanced effects allowed by various constraints: one from holomorphic trilinear soft SUSY breaking terms and/or right handed up squark mass insertions and another from non-holomorphic trilinear soft SUSY breaking terms and light Higgs boson masses. Interestingly, even with $\mathcal{O}(1)$ flavour violating effects in the, presently unconstrained, up-squark sector, SUSY effects on $\mathcal{B}(t \rightarrow q h)$ come out to be unobservable at LHC mainly due to leading order cancellations between penguin and self energy diagrams and the constraints from charge- and colour-breaking minima (CCB) of the MSSM vacuum. An exception to this conclusion may be effects arising from non-holomorphic soft SUSY breaking terms in the region where the CP-odd Higgs mass is smaller than the top-quark mass but this scenario is disfavoured by recent LHC searches. Our calculations for $t \rightarrow q h$ decay are made available in SUSY_FLAVOUR numerical library.

Keywords: Supersymmetry Phenomenology

ARXIV EPRINT: 1409.6546 


\section{Contents}

1 Introduction 1

2 Calculation of $\mathcal{B}(t \rightarrow q h)$ in MSSM

2.1 Branching ratio and QCD corrections 6

$\begin{array}{lll}2.2 & \text { Wilson coefficients: full MSSM corrections } & 7\end{array}$

$3 \mathcal{B}(t \rightarrow q h):$ cancellations, decoupling and qualitative results 9

4 Constraints from other observables 14

5 Results $\quad \mathbf{1 5}$

5.1 Enhancement through large $\frac{\left|A_{U}^{3 I}\right|}{M_{S}}$ and $\frac{\left|A_{U}^{I 3}\right|}{M_{S}} \quad 16$

5.2 The light $M_{A}$ scenario and non-holomorphic dominance 20

6 Conclusions $\quad 22$

$\begin{array}{ll}\text { A Explicit expressions for MSSM vertices } & 23\end{array}$

B Passarino-Veltman loop functions $\quad 25$

\section{Introduction}

The last fundamental elementary particles discovered during the last 20 years are the tau-neutrino by DONUT Collaboration [1], the top quark at Tevatron [2,3] with mass $m_{t}=172.5 \mathrm{GeV}$ and the Higgs boson [4-6] at LHC [7, 8], with mass $m_{h} \approx 126 \mathrm{GeV}$. Among them, the top quark has been and will be produced in large numbers at LHC, allowing for increasingly accurate measurements of its properties. LHC operating at c.m. energy of 7 and $8 \mathrm{TeV}$ has already collected about two-million $t \bar{t}$-pairs. It is therefore timely to examine the possibility of rare, flavour-changing (FC), top decays to the light up-quarks, $u$ or $c$, and the Higgs boson $h$,

$$
t \rightarrow u h, \quad \text { or } t \rightarrow c h .
$$

We collectively denote these processes as $t \rightarrow q h$ with $q=u, c$. The Higgs boson field $h$ is understood as one of the possible scalar fields that couples to up-quarks and has mass smaller than that of the top-quark.

If the decays $t \rightarrow q h$ are governed only by the Standard Model (SM) [9] dynamics they would never be observed at LHC because their branching ratios, $\mathcal{B}(t \rightarrow u h)_{\mathrm{SM}} \approx 4 \times 10^{-17}$ and $\mathcal{B}(t \rightarrow c h)_{\mathrm{SM}} \approx 4 \times 10^{-14}[10,11]$, are tiny. This extraordinary suppression is caused because, firstly, the Glashow-Iliopoulos-Maiani (GIM) [12] suppression prohibits the loop 
diagram leading contribution for $t \rightarrow q h$, and secondly, because the quarks circulating in the $t \rightarrow q h$ loop amplitude are those of down type with small mass differences.

On the contrary, in a well motivated extension of the SM, the R-parity conserving Minimal Supersymmetric Standard Model (MSSM) [13-15], although the GIM mechanism is still operative in the quark-interactions, it is not, in general, in the squark interactions. Eventually, coloured scalars, the squarks, enter in loops with potentially large mass differences. The question is then whether these new interactions are able to enhance $\mathcal{B}(t \rightarrow q h)$ up to an observable level at LHC. Depending on MSSM input parameters, Guasch and Sola [16] arrived at a maximum prediction $\mathcal{B}(t \rightarrow c h) \approx 4 \times 10^{-4}$, while a more recent analysis by Cao et al. $[17,18]$, taking into account constraints from rare $B$-meson decays, concluded a maximum branching fraction of up to $\mathcal{B}(t \rightarrow c h) \approx 6 \times 10^{-5}$ (for an earlier study see also [19]). Finally, not long ago, a new analysis by the authors of [20] concluded a maximum branching ratio at the level of $O\left(10^{-6}\right)$ after constraints.

The relevant Lagrangian governing the rare top decays $t \rightarrow q h$ in the physical quark basis, after integrating out all heavy degrees of freedom, is simply,

$$
-\mathcal{L} \supset C_{L}^{(h)} \bar{q}_{R} t_{L} h+C_{R}^{(h)} \bar{q}_{L} t_{R} h+\text { H.c }
$$

with dimensionless (Wilson) coefficients $C_{L, R}^{(h)}$. Note that in the MSSM $h$ may stand for one of the two CP-even Higgs bosons denoted as $h, H$, respectively. Currently LHC sets an upper bound [21, 22]

$$
\mathcal{B}(t \rightarrow q h) \leq 0.79 \%(\mathrm{ATLAS}), \quad \mathcal{B}(t \rightarrow q h) \leq 0.56 \%(\mathrm{CMS})
$$

This result places rather weak restrictions onto the Wilson coefficients: $\left|C_{L}\right|,\left|C_{R}\right| \lesssim 0.1$. In renormalisable theories like the MSSM, the coefficients $C_{L}$ and $C_{R}$ would come from one-loop diagrams involving gluino (or neutralino)-up squarks, chargino-down squarks and charged Higgs-down quarks. The gluino-loop gives the dominant contribution to $\mathcal{B}(t \rightarrow q h)$ that generically is of the order $\alpha_{s} / 4 \pi \approx 0.01$, which is by an order of magnitude less than the current bound, but probably within LHC's projected reach at $\sqrt{s}=14 \mathrm{TeV}$ with 3000 $\mathrm{fb}^{-1}[23]$ (see also note [24])

$$
\mathcal{B}(t \rightarrow q h) \lesssim 2.0 \times 10^{-4} \Leftrightarrow\left|C_{L}\right|,\left|C_{R}\right| \lesssim \mathcal{O}(1) \times 10^{-2}
$$

There are already many phenomenological studies for these decays, a partial list included in [25-32]. Very recently in [33, 34], plausible techniques that distinguish between $t \rightarrow u h$ and $t \rightarrow c h$ have been suggested. It is therefore worth looking for MSSM branching fraction predictions from both rare top decays, $t \rightarrow u h$ and $t \rightarrow c h$.

The new flavour structure in the MSSM Lagrangian can be parametrized in terms of supersymmetry soft breaking squark mass matrices $m_{Q_{L}}, m_{U_{R}}, m_{D_{R}}$ and trilinear holomor- 
phic $A_{U}, A_{D}$ matrices as well as the trilinear non-holomorphic $A_{U}^{\prime}, A_{D}^{\prime}$ matrices $[35-38]^{1}$

$$
\begin{aligned}
\mathcal{L}_{\mathrm{MSSM}} \supset & -\widetilde{Q}_{L}^{\dagger} m_{Q_{L}}^{2} \widetilde{Q}_{L}-\widetilde{U}_{R}^{\dagger} m_{U_{R}}^{2} \widetilde{U}_{R}-\widetilde{D}_{R}^{\dagger} m_{D_{R}}^{2} \widetilde{D}_{R} \\
& +\left(H_{2} \widetilde{Q}_{L} A_{U} \widetilde{U}_{R}+H_{1} \widetilde{Q}_{L} A_{D} \widetilde{D}_{R}+\text { H.c }\right) \\
& +\left(H_{1}^{\dagger} \widetilde{Q}_{L} A_{U}^{\prime} \widetilde{U}_{R}+H_{2}^{\dagger} \widetilde{Q}_{L} A_{D}^{\prime} \widetilde{D}_{R}+\text { H.c }\right),
\end{aligned}
$$

where flavour and gauge group indices have been suppressed. As we already mentioned, soft breaking terms in (1.5) may have non-trivial structure, so that the quark and squark mass matrices cannot be diagonalized simultaneously in the same flavour basis. However, a fully generic structure for these matrices is far excluded by Kaon, charm, and $B$-physics experiments with the exception of the right handed up-squark mass matrix $m_{U_{R}}^{2}$ and the trilinear soft SUSY breaking matrices $A_{U}$ and $A_{U}^{\prime}$. For all other matrices $m$ and $A$ in (1.5), "flavour" experiments help to single out four possible categories:

1. Minimal Flavour Violation (MFV) assumption [36, 39]: flavour violation arises only from Yukawa matrices $Y_{U}$ and $Y_{D}$.

2. Almost degenerate $m$ 's - their diagonal elements proportional to the unit matrix; $A$ 's almost diagonal; small off-diagonal terms in $m$ 's and $A$ 's.

3. As in point (2) but $m$ 's become hierarchical: 1st and 2nd generation are much heavier than the third. In this case off-diagonal squark mass matrix elements may be of order one.

4. Alignment: no particular hierarchy among diagonal squark masses, but small squark mixing angles, enforced by some symmetries, as required by experimental constraints.

MFV basically leads to the same suppression pattern for $t \rightarrow q h$ as in the SM and therefore no signal observation is expected at LHC [40]. ${ }^{2}$ We need therefore to depart from MFV. This is most conveniently done by considering the dimensionless flavour violating expansion parameters (commonly called "mass insertions") [41, 42]:

$$
\Delta_{\tilde{X}}^{I J}=\frac{\left(m_{X}^{2}\right)^{I J}}{\sqrt{\left(m_{X}^{2}\right)^{I I}\left(m_{X}^{2}\right)^{J J}}},
$$

which denotes the ratio of flavour-violating squark mass matrix elements over an average of flavour-conserving squark mass matrix elements $(\tilde{X}$ can be $\tilde{U}$ or $\tilde{D})$. It has been shown

\footnotetext{
${ }^{1}$ Non-holomorphic terms may arise from the Kähler potential non-renormalizable operators like for example $X X^{\dagger} H_{1}^{\dagger} Q_{L} U_{R} / M^{3}$ interaction between MSSM superfields and hidden sector superfield $X$ whose F-term vev, $\left\langle F_{X}\right\rangle$, is responsible for spontaneous SUSY breaking in the hidden sector. In contrast, the holomorphic SUSY breaking terms arise from superpotential non-renormalizable operators like, $X H_{2} Q_{L} U_{R} / M$. If SUSY breaking mediators of mass $\mathcal{O}(M)$ are very heavy, as for instance in gravity mediated SUSY breaking scenario where $M=M_{P l}$, then non-holomorphic terms $\left(A_{U}^{\prime}\right)$ are negligible compared to the holomorphic ones $\left(A_{U}\right)$. However, they could both be of the same order of magnitude if SUSY breaking happens at low SUSY breaking scales, comparable to electroweak scale [37].

${ }^{2}$ This is also due to the fact that no $\tan \beta$ enhanced top flavour changing decay amplitudes arise in the MSSM as we will see shortly.
} 
in ref. [43] that, for $\Delta F=1$ processes, the same (in magnitude) $\Delta$-parameter can be used to parametrize flavour effects in both cases of hierarchical and degenerate squark masses, although the $\Delta$-parameter may have different meaning in each case. We develop a similar technique here in expanding the full amplitude for $t \rightarrow q h$ in powers of $\Delta$ 's and therefore discussing cases (2) and (3) in a unified way.

In the fourth case of alignment quark and squark mass matrices are forced by some approximate flavour symmetry to be diagonalized almost by the same field rotation. This means that the remaining squark rotation angles in the super-CKM basis are small, but in general, squark masses are far from degenerate leading to serious constraints from Kphysics. In any case, having the light Higgs boson mass at $126 \mathrm{GeV}$, one needs pushing the stop mixing angle to the maximal value. This situation does not fit naturally to the case of small mixing angles. On this ground we will not examine this case.

In fact we shall show below that the LHC projected bound (1.4) is impossible to be reached in the general R-parity conserved MSSM with degenerate or hierarchical squark mass spectrum. This is partly due to cancellations between self energy and penguin contributions prohibiting non-decoupling SUSY effects. As a result, in the best case scenario, and before constraints, an estimate of the dominant gluino-squark diagrams results in

$$
C_{L, R}^{(h)} \approx \frac{\alpha_{s}}{4 \pi}\left(\frac{m_{t}}{M_{S}}\right)^{2} \Delta \lesssim 2 \times 10^{-4}
$$

for degenerate SUSY squark masses $M_{S}$ at $1 \mathrm{TeV}$ scale and $\Delta=\mathcal{O}(1)$. Similar cancellations exist in the chargino-squark loops but now $\alpha_{s} \rightarrow \alpha_{2}$ and therefore, following (1.7), $C_{L, R}^{(h)}$ are by at least a factor of three smaller than the gluino contribution. ${ }^{3}$ Furthermore, as it is obvious from (1.7), both our analytical and numerical study concludes that there are no non-decoupling effects whatsoever for large SUSY mass spectrum, collectively indicated here as $M_{S}$.

To the best of our knowledge, this study deals with four new aspects of $\mathcal{B}(t \rightarrow q h)$ not considered before in the literature [16-20]:

1. We take into account the effects of Next to Leading order QCD corrections due to the SUSY loop induced chromomagnetic dipole operator and the running of operators from the SUSY scale $M_{S}$ to the top quark scale (see section 2).

2. We present analytical details of the cancellations and decoupling (section 3), using a common scheme for both universal and hierarchical squark mass structures.

3. We investigate the effect on $\mathcal{B}(t \rightarrow q h)$ from non-holomorphic SUSY breaking terms $A_{U}^{\prime}$ [see eq. (1.5)] (section 5).

4. Finally, we have encoded all our calculations into a publicly available ${ }^{4}$ SUSY_FLAVOUR library [44-46]. SUSY_FLAVOUR uses the relevant and most complete up-to-date constraints from FCNC processes (section 4).

\footnotetext{
${ }^{3}$ In fact chargino diagrams are far smaller than that because of the down squark circulation in loop. The relevant $\Delta_{\tilde{D}}$ 's in this case must be small to respect experimental constraints from low energy meson experiments. Similar situation applies to charged Higgs boson one-loop diagrams.

${ }^{4}$ SUSY_FLAVOUR can be downloaded from http://www.fuw.edu.pl/susy_flavor
} 


\section{Calculation of $\mathcal{B}(t \rightarrow q h)$ in MSSM}

The gauge-invariant dimension-6 operator responsible for the decay $t \rightarrow q h$ can be, after decoupling of heavy particles, simply written $\operatorname{as}^{5}$

$$
O^{(h)}=\left(H^{\dagger} H\right) \bar{Q}_{L}^{I} u_{R}^{J} \widetilde{H}+\text { H.c. }
$$

$H$ is the SM Higgs field $\mathrm{SU}(2)$ doublet, $\widetilde{H}=i \sigma_{2} H^{*}$ is its charged conjugate, indices $I$ and $J$ denote quark flavours, $Q_{L}^{I}$ is the left-handed quark $\mathrm{SU}(2)$ doublet while $u_{R}^{J}$ is the right-handed up-quark singlet. $\mathrm{SU}(2)$ and $\mathrm{SU}(3)$ indices are not shown explicitly. The effective operator in $O^{(h)}$ is of (pseudo)-scalar form and affects the renormalizable Yukawa interaction $\bar{Q}_{L}^{I} u_{R}^{J} \widetilde{H}$. After electroweak symmetry breaking (EWSB) it results in the effective Lagrangian (1.2).

It was shown recently in ref. [47] that the operator $O^{(h)}$ mixes through QCD strong interactions with the gluonic dipole operator that has the form

$$
O^{(g)}=g_{s} \bar{Q}_{L}^{I} \sigma^{\mu \nu} \lambda^{A} u_{R}^{J} \widetilde{H} G_{\mu \nu}^{A}+\text { H.c. }
$$

where $g_{s}=\sqrt{4 \pi \alpha_{s}}$ is the strong QCD coupling, $\lambda^{A}$ are the Gell-Mann matrices, while $G_{\mu \nu}^{A}$ is the $\mathrm{SU}(3)$ field strength tensor. Like the operator $O^{(h)}$, the operator $O^{(g)}$ is also chirality flipping. After EWSB it results in the effective Lagrangian term

$$
-\mathcal{L} \supset C_{L}^{(g) I J} \bar{u}_{R}^{I} \sigma_{\mu \nu} \lambda^{A} u_{L}^{J} G^{A \mu \nu}+C_{R}^{(g) I J} \bar{u}_{L}^{I} \sigma_{\mu \nu} \lambda^{A} u_{R}^{J} G^{A \mu \nu}+\text { H.c. }
$$

Having listed all operators needed, we enumerate here our steps in calculating $\mathcal{B}(t \rightarrow q h):$

1. Full calculation of the relevant 1-Particle-Irreducible (1PI) Feynman diagrams $C_{L, R}^{(h)}$ at scale $M_{S}$, where $M_{S}$ is the lightest coloured sparticle (squark or gluino) mass.

2. Full calculation of the SUSY induced Wilson coefficient $C_{L, R}^{(g)}$ associated with the dipole operator $O^{(g)}$ that mix with strong (QCD) quantum corrections.

3. Use Renormalization Group Equations (RGEs) with formulae taken from [47] to run all operators down to the top mass scale.

4. Calculate the branching fraction at $m_{t}^{\text {pole }}$.

In the next two subsections we append technical details entailed in these steps.

\footnotetext{
${ }^{5}$ In full SUSY limit with all Higgs (super)fields present, the corresponding operator is an F-term and, therefore, holomorphic. It has the form $O^{(h)}=\left(H_{1} H_{2}\right) Q_{L}^{I} u_{R}^{J} H_{2}+$ H.c . Note that this operator breaks Peccei-Quin and R-symmetry invariance and therefore its Wilson coefficient must be proportional to quantities that violate these symmetries, such as the gluino mass, the trilinear soft SUSY breaking couplings and the $\mu$-parameter, c.f. eq. (3.9).
} 


\subsection{Branching ratio and QCD corrections}

In this section we present the calculation for the decay of the top quark into a light quark $q=u, c$ and a CP-even Higgs boson $h \equiv(H$ or $h)$ including NLO QCD corrections. In the limit $m_{q} \approx 0$ the tree level decay rate reads: ${ }^{6}$

$$
\Gamma_{0}(t \rightarrow q h)=\frac{m_{t}}{32 \pi}\left(\left|C_{L}^{(h)}\right|^{2}+\left|C_{R}^{(h)}\right|^{2}\right)\left(1-\frac{m_{h}^{2}}{m_{t}^{2}}\right)^{2},
$$

with $C_{L, R}^{(h)}$ defined in eq. (1.2). At the top-quark mass scale, $\mu=m_{t}$, the following QCD NLO decay rate is found [47],

$$
\Gamma(t \rightarrow q h)=1.018 \Gamma_{0}+0.049 \frac{m_{t}^{3}}{16 \pi v}\left(1-\frac{m_{h}^{2}}{m_{t}^{2}}\right)^{2} \Re e\left[C_{R}^{(h) *} C_{R}^{(g)}+C_{L}^{(h) *} C_{L}^{(g)}\right],
$$

with $C_{L(R)}^{(g)}$ defined in eq. (2.3). We use $\alpha_{s}\left(m_{t}\right)=0.1079, m_{t}\left(m_{t}\right)_{\overline{D R}}=163.6 \mathrm{GeV}, m_{t}^{\text {pole }}=$ $172.5 \mathrm{GeV}, G_{F}=1 / \sqrt{2} v^{2}=1.1664 \times 10^{-5} \mathrm{GeV}^{-2}$. In our results we have neglected terms proportional to $\left|C_{L, R}^{(g)}\right|^{2}$ since they are small for $m_{h} \simeq 126 \mathrm{GeV}$. For the branching fraction $\mathcal{B}(t \rightarrow q h)$, the next-to-next-to-leading order top quark width is used, $\Gamma(t \rightarrow b W)=$ $1.39 \mathrm{GeV}$ [48]. Furthermore, we assume that the "tree level" decay width $\Gamma(t \rightarrow b W)$ is not affected substantially by SUSY loop contributions. In this section, we calculate the Wilson coefficients, $C_{L, R}^{(h)}$ and $C_{L, R}^{(g)}$, at the scale $\mu=M_{\mathrm{S}}=m_{\tilde{g}}$, and use the renormalization group equations [47] to run them down to the scale $\mu=m_{t}$,

$$
\begin{aligned}
C_{L, R}^{(h)}\left(m_{t}\right)= & C_{L, R}^{(h)}\left(M_{\mathrm{S}}\right)\left(\frac{\alpha_{s}\left(M_{\mathrm{S}}\right)}{\alpha_{s}\left(m_{t}\right)}\right)^{-4 / b_{3}} \\
& +\frac{24}{7} \frac{m_{t}\left(m_{t}\right)^{2}}{v} C_{L, R}^{(g)}\left(M_{\mathrm{S}}\right)\left[\left(\frac{\alpha_{s}\left(M_{\mathrm{S}}\right)}{\alpha_{s}\left(m_{t}\right)}\right)^{2 /\left(3 b_{3}\right)}-\left(\frac{\alpha_{s}\left(M_{\mathrm{S}}\right)}{\alpha_{s}\left(m_{t}\right)}\right)^{-4 / b_{3}}\right], \\
C_{L, R}^{(g)}\left(m_{t}\right)= & C_{L, R}^{(g)}\left(M_{\mathrm{S}}\right)\left(\frac{\alpha_{s}\left(M_{\mathrm{S}}\right)}{\alpha_{s}\left(m_{t}\right)}\right)^{2 /\left(3 b_{3}\right)}
\end{aligned}
$$

where $b_{3}=11-2 N_{f} / 3$ is the 1 -loop gluon $\beta$-function. In our case $N_{f}=6$, i.e., we assume there are no other coloured particles below $M_{\mathrm{S}}$ except from the six SM quark flavours. Diagrams that do not involve coloured particles are "frozen" at the $m_{t}$-scale and do not participate in the running of Wilson coefficients in eq. (2.6b).

It turns out that the effect of consistently including NLO QCD corrections in $\mathcal{B}(t \rightarrow q h)$ is about $20 \%$. This is primarily due to the RGE running of $C_{L, R}^{(h)}$ from $M_{\mathrm{S}}$ down to the top quark mass scale, and, secondarily due to finite SUSY corrections in $C^{(g)}$ present in the decay width (2.5). The $C^{(h)}$ and $C^{(g)}$ coefficients, although in theory different in their Dirac and Lorentz structures, are both subject to the same squark-gluino Feynman diagram contribution. $C^{(g)}$ has analogous, and even more persisting, cancellations than $C^{(h)}$, due to the flavour conserving gluon-squark vertex of the former, and the same flavour changing

\footnotetext{
${ }^{6}$ Although straightforward, decays $t \rightarrow q A$ with $A$ being the CP-odd Higgs boson are only marginally permitted by recent LHC data and therefore not considered in this work, c.f., discussion in section 5.2.
} 


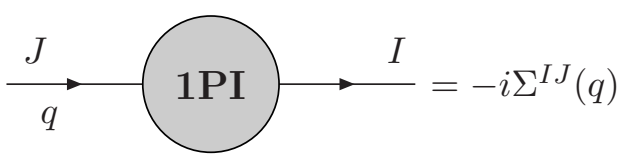

(a)

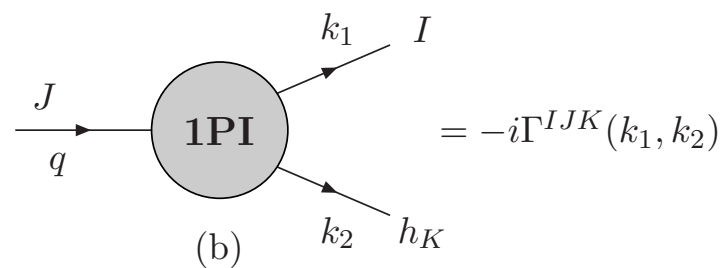

(b)

Figure 1. (a) Quark self energy one-particle irreducible (1PI) diagram corrections. (b) 1PI penguin contribution to $u^{J} \rightarrow u^{I}+h_{K}$.

insertions i.e., same $\Delta$ 's. As a result, it turns out that SUSY contributions to $m_{t} C^{(g)}$ are at most of the same order as in $C^{(h)}$ and give an amount of $2-10 \%$ correction to the decay width.

\subsection{Wilson coefficients: full MSSM corrections}

Expressions for Wilson-coefficients are more transparent if we write them in terms of the one particle irreducible (1PI) diagrams for self energies $(\Sigma)$ and penguins $(\Delta F)$, as in figure 1. We define:

$$
\begin{aligned}
\Sigma^{I J}(p) & =\Sigma_{V L}^{I J}\left(p^{2}\right) \not p P_{L}+\Sigma_{V R}^{I J}\left(p^{2}\right) \not p P_{R}+\Sigma_{m L}^{I J}\left(p^{2}\right) P_{L}+\Sigma_{m R}^{I J}\left(p^{2}\right) P_{R}, \\
\Gamma^{I J K}\left(k_{1}, k_{2}\right) & =\Delta F_{L}^{I J K}\left(k_{1}, k_{2}\right) P_{L}+\Delta F_{R}^{I J K}\left(k_{1}, k_{2}\right) P_{R} .
\end{aligned}
$$

All $\Sigma$ 's and $\Delta F$ 's depend on external momenta and internal masses. We follow everywhere the Feynman rules, notation and conventions, from refs. [49, 50]. For specific processes, the top-quark is identified with $J=3$ and the charm-(up-)quark with $I=2(I=1)$, the "little $h$ " Higgs boson with $K=2$, the "big $H$ " with $K=1$, but otherwise we keep the $I, J$ and $K$ notation as general as possible.

Using standard on-shell renormalization scheme techniques we obtain for $I \neq J$ :

$$
\begin{aligned}
C_{L}^{(h) I J K}= & \frac{\eta^{K}}{m_{J}^{2}-m_{I}^{2}}\left\{m_{I} m_{J}^{2}\left[\Sigma_{V L}^{I J}\left(m_{J}^{2}\right)-\Sigma_{V L}^{I J}\left(m_{I}^{2}\right)\right]+m_{I}^{2} m_{J}\left[\Sigma_{V R}^{I J}\left(m_{J}^{2}\right)-\Sigma_{V R}^{I J}\left(m_{I}^{2}\right)\right]\right. \\
& \left.+m_{I} m_{J}\left[\Sigma_{m R}^{I J}\left(m_{J}^{2}\right)-\Sigma_{m R}^{I J}\left(m_{I}^{2}\right)\right]+\left[m_{I}^{2} \Sigma_{m L}^{I J}\left(m_{J}^{2}\right)-m_{J}^{2} \Sigma_{m L}^{I J}\left(m_{I}^{2}\right)\right]\right\} \\
& +\left(\Delta F_{L}\right)^{I J K}
\end{aligned}
$$

and $C_{R}=C_{L}:(L \leftrightarrow R)$. The parameter $\eta$ is defined as $\eta^{K} \equiv Z_{R}^{2 K} / v_{2}$ with $Z_{R}$ defined in (A.1). The self energy components obey the following hermicity conditions $\Sigma_{V L(R)}^{J I \star}=$ $\Sigma_{V L(R)}^{I J}$ and $\Sigma_{m L(R)}^{J I \star}=\Sigma_{m R(L)}^{I J}$ and explicitly read in a most compact notation as $(S=$ scalar, $F=$ fermion):

$$
\begin{aligned}
\Sigma_{V L}^{I J}[p, S, F] & \equiv \sum_{i, j} V_{u S F, L}^{I j i *} V_{u S F, L}^{J j i}\left(B_{1}+B_{0}\right)\left[p, m_{S_{j}}, m_{F_{i}}\right] \\
\Sigma_{m L}^{I J}[p, S, F] & \equiv \sum_{i, j} m_{F_{i}} V_{u S F, R}^{I j i *} V_{u S F, L}^{J j i} B_{0}\left[p, m_{S_{j}}, m_{F_{i}}\right]
\end{aligned}
$$


with $L \leftrightarrow R$ for $\Sigma_{V R}^{I J}$ and $\Sigma_{m R}^{I J}$. Generic vertices $V_{u S F}$ follow the notation of appendix A.2 in ref. [51]. Explicitly for individual SUSY particles, their forms, copied from ref. [50], are given in A for complementarity. Detailed definitions for two-point one-loop functions $B_{0}, B_{1}$ are given in $\mathrm{B}$.

The SUSY-mediated $t \rightarrow q h$ penguin amplitudes can be classified into two distinct topologies: (SFS) squark-gluino/neutralino/chargino-squark and (FSF) chargino/neutralino-squark-chargino/neutralino vertex diagrams. They both contribute to the expressions for the $C_{L, R}^{(h)}$ in (1.2),

$$
\Delta F_{L}=\Delta F_{L}^{(S F S)}+\Delta F_{L}^{(F S F)}
$$

where in a self-explanatory notation

$$
\begin{aligned}
& \Delta F_{L}^{(S F S)}=\Delta F_{L}^{(\tilde{D} \chi \tilde{D})}+\Delta F_{L}^{\left(\tilde{U} \chi^{0} \tilde{U}\right)}+\Delta F_{L}^{(\tilde{U} \tilde{g} \tilde{U})}, \\
& \Delta F_{L}^{(F S F)}=\Delta F_{L}^{(\chi \tilde{D} \chi)}+\Delta F_{L}^{x\left(\chi^{0} \tilde{U} \chi^{0}\right)},
\end{aligned}
$$

and similar for $\Delta F_{R}^{(S F S, F S F)}$ with the substitution $L \leftrightarrow R$. Each term in the above expressions will be given by a straightforward substitution in the following compact forms (again explicit vertices for the generalised $V$ 's as well as integral functions can be found in the appendices.) External momenta follow the conventions of figure 1b:

$$
\begin{aligned}
\Delta F_{L}^{(S F S) I J K}=- & \sum_{i, j, l}\left\{m_{I}\left(V_{H S S}^{K l i} V_{u S F, L}^{I l j *} V_{u S F, L}^{J i j}\right)\left(C_{12}-C_{11}\right)\right. \\
& +m_{J}\left(V_{H S S}^{K l i} V_{u S F, R}^{I l j *} V_{u S F, R}^{J i j}\right)\left(C_{11}+C_{0}\right) \\
& \left.+m_{F_{j}}\left(V_{H S S}^{K l i} V_{u S F, R}^{I l j *} V_{u S F, L}^{J i j}\right) C_{0}\right\}\left[k_{2}, k_{1}, m_{S_{i}}, m_{S_{l}}, m_{F_{j}}\right] \\
\Delta F_{L}^{(F S F) I J K}=- & \sum_{i, j, l}\left\{\left(V_{u S F, R}^{I j l *} V_{F H F, R}^{i K l} V_{u S F, L}^{J j i}\right)\left(\tilde{C}_{0}+m_{I}^{2} C_{11}+\left(m_{J}^{2}-m_{I}^{2}\right) C_{12}\right)\right. \\
& +m_{I} m_{J}\left(V_{u S F, L}^{I j l *} V_{F H F, L}^{i K l} V_{u S F, R}^{J j i}\right)\left(C_{0}+C_{11}\right) \\
& +m_{F_{l}} m_{F_{i}}\left(V_{u S F, R}^{I j l *} V_{F H F, L}^{i K l} V_{u S F, L}^{J j i}\right) C_{0} \\
& +m_{I} m_{F_{i}}\left(V_{u S F, L}^{I j l *} V_{F H F, L}^{i K l} V_{u S F, L}^{J j i}\right)\left(C_{0}+C_{11}-C_{12}\right) \\
& +m_{J} m_{F_{i}}\left(V_{u S F, R}^{I j l *} V_{F H F, R}^{i K L} V_{u S F, R}^{J j i}\right) C_{12} \\
& +m_{I} m_{F_{l}}\left(V_{u S F, L}^{I j l *} V_{F H F, R}^{i K l} V_{u S F, L}^{J j i}\right)\left(C_{11}-C_{12}\right) \\
& \left.+m_{J} m_{F_{l}}\left(V_{u S F, R}^{I j l *} V_{F H F, L}^{i K l} V_{u S F, R}^{J j i}\right)\left(C_{0}+C_{12}\right)\right\}\left[k_{1}, k_{2}, m_{S_{j}}, m_{F_{l}}, m_{F_{i}}\right] .
\end{aligned}
$$

Again, from these expressions one may also derive the corresponding $\Delta F_{R}^{(S F S, F S F)}$ by just letting $L \leftrightarrow R$. Integral functions and vertices are given in A and B. We have checked both analytically and numerically that the SUSY contributions to $t \rightarrow q h$ amplitudes $C_{L, R}$ are finite and renormalization scale invariant. For our numerical analysis, we have included all the above full expressions into the SUSY_FLAVOUR library.

Note that a calculation of the effective Higgs-quark vertices in the MSSM, however without detailed analysis of their phenomenological implications for top quark decays, can 
also be found in refs. $[52,53]$. As discussed there, effects of resummation from higher order chiral corrections are small in the up-quark sector and should not change our qualitative discussion below. However, as such corrections are implemented in SUSY_FLAVOUR library, they can indirectly affect those bounds on the flavour changing up-squark $\Delta$ parameters which are given by measurements of processes involving down quarks but sensitive to upsquarks circulating in loop amplitudes.

\section{$3 \mathcal{B}(t \rightarrow q h)$ : cancellations, decoupling and qualitative results}

The formulae given previously, although most general, are quite opaque and do not allow for, at least qualitative, discussion of possible cancellation or enhancement effects taking place in coefficients $C_{L, R}^{(h)}$ of eq. (1.2). We therefore need to perform some approximations.

In the limit where $m_{I}=m_{u}\left(m_{c}\right) \rightarrow 0$, the coefficients in (2.9) can be written simply as $(I=1,2, J=3)$,

$$
\begin{aligned}
C_{L}^{(h) I J} & =\Delta F_{L}^{(h) I J}-\frac{1}{v}\left(\frac{\cos \alpha}{\sin \beta}\right) \Sigma_{m L}^{I J}(0), \\
C_{L}^{(H) I J} & =\Delta F_{L}^{(H) I J}-\frac{1}{v}\left(\frac{\sin \alpha}{\sin \beta}\right) \Sigma_{m L}^{I J}(0),
\end{aligned}
$$

with an obvious substitution $L \leftrightarrow R$ for the coefficients $C_{R}^{(h)}, C_{R}^{(H)}$. The coefficients that multiply the self energy 1PI diagrams are not simply proportional to $\tan \beta$ as for example is the case for the $\bar{b} s h$ transitions in the MSSM. In the SM limit, where the CP-odd Higgs mass $M_{A}$ (and therefore the CP-even Higgs boson mass $M_{H}$ ) is taken to be much heavier than $M_{Z}$, we have [54]

$$
M_{A} \gg M_{Z} \quad: \quad \cos \alpha \approx \sin \beta, \quad \sin \alpha \approx-\cos \beta .
$$

In this case only the decay $t \rightarrow q h$ (i.e., $K=2$ ) is relevant and the amplitude is

$$
C_{L}^{(h) I J} \stackrel{\mathrm{SM}}{=} \Delta F_{L}^{(h) I J}-\frac{1}{v} \Sigma_{m L}^{I J}(0)
$$

with an analogous formula for $C_{R}^{(h)}{ }^{7}$ In our analytical results for $t \rightarrow q h$ amplitude below, we shall work with the general expressions in eqs. (3.1) and (3.2) and take the SM-limit (3.3) when necessary.

Due to the presence of the strong QCD coupling, gluino diagrams are expected to be dominant. Their contributions can be deduced easily from the general expressions in

\footnotetext{
${ }^{7}$ At the moment, LHC data cannot completely exclude [55] but rather disfavour [56] the existence of more than one Higgs boson lighter than $m_{t}$, with such scenario limited only to certain "tuned" scenarios (see for example ref. [57]).
} 
eqs. (2.10b) and (2.13a) and give

$$
\begin{aligned}
C_{L}^{\left(h_{K}\right) I J}= & -\frac{2 \alpha_{s}}{3 \pi}\left(\frac{m_{t}}{m_{\tilde{g}}^{2}}\right) \sum_{i, l=1}^{6} V_{H U U}^{K l i} Z_{U}^{(J+3) i *} Z_{U}^{(I+3) l}\left(C_{0}+C_{11}\right)\left[\kappa_{2}, \kappa_{1}, r_{i}, r_{l}, 1\right] \\
& +\frac{2 \alpha_{s}}{3 \pi}\left(\frac{1}{m_{\tilde{g}}}\right) \sum_{i, l=1}^{6} V_{H U U}^{K l i} Z_{U}^{J i *} Z_{U}^{(I+3) l} C_{0}\left[\kappa_{2}, \kappa_{1}, r_{i}, r_{l}, 1\right] \\
& +\frac{2 \alpha_{s}}{3 \pi}\left(\frac{Z_{R}^{2 K}}{v_{2}}\right) m_{\tilde{g}} \sum_{i=1}^{6} Z_{U}^{J i *} Z_{U}^{(I+3) i} B_{0}\left[0, r_{i}, 1\right]
\end{aligned}
$$

where the first two $\left(\Delta F_{L}\right)$ contributions arise from the gluino penguin with flipped chirality in the top quark external line and the gluino internal line respectively, while the last from the self energy $\left(\Sigma_{m L}\right)$, gluino diagram. The symbols in (3.5) are defined in A. In particular, the mass dimension one, Higgs-squark vertex, $V_{H U U}^{K l i}$, can be read explicitly from (A.4) and $Z_{U}$ is the unitary matrix diagonalizing the up-squark mass matrix (see (A.2)), in the basis where quarks are diagonal. ${ }^{8}$ Finally, in (3.5), we have changed to a more suggestive form of Passarino-Veltman (PV) functions with dimensionless parameters, $\kappa_{i} \equiv k_{i} / m_{\tilde{g}}, r_{i} \equiv m_{i}^{2} / m_{\tilde{g}}^{2}$, by simply factoring out the gluino mass scale (details of the transformation along with useful properties of the PV functions can be found in B). Note that in the completely universal case (MFV scenario) where $r_{i}=$ const, the whole gluino contribution (3.5) vanishes identically due to unitarity of the $Z_{U}$-matrices.

It is interesting to check (3.5) for non-decoupling effects. As we can see from (A.4), the vertex behaves at most as $V_{H U U} \sim M_{S}$ and therefore, individually, the last two terms in (3.5), do not decouple separately when all SUSY parameters are scaled up by the same factor. However, this non-decoupling behaviour is not realised because of partial cancellations between the penguin and self energy contributions given in (3.5b) and (3.5c), respectively. More specifically, potentially non-decoupled contributions cancel among each other leaving behind remnants with $\sim m_{t}^{2} / M_{S}^{2}$ as leading behaviour. In this section, we will show this behaviour both numerically, in the full expression, and analytically, up to a certain order in the relevant expansion. For the following quantitative analysis of cancellations and the leading order contributions, it is sufficient to work in the zero external momentum approximation for the penguin and self energy diagrams.

Before proving the cancellations and estimating the behaviour of surviving contributions, we open a parenthesis here to present a useful theorem from matrix algebra. It says the following: consider a Hermitian $n \times n$ matrix $A$. The trivial decomposition $A=A^{0}+\widetilde{A}$, where $A^{0}=\operatorname{diag}\left(a_{1}^{0}, a_{2}^{0}, \ldots, a_{n}^{0}\right)$ contains the diagonal elements of $A$ and $\widetilde{A}$ contains the non-diagonal elements of $A$, is always possible. Let the unitary matrix $U$ diagonalizes the matrix $A$ as $U^{\dagger} A U=D$, where $D=\operatorname{diag}\left(d_{1}, d_{2}, \ldots, d_{n}\right)$ is a diagonal matrix containing the eigenvalues of matrix $A$. If we assume that $f$ is an arbitrary analytic function, we can

\footnotetext{
${ }^{8}$ For more details on the exact definitions of the squark mass and rotation matrices the reader is referred to ref. [50].
} 
write down the following decomposition of matrix $f(A)$ in powers of $\widetilde{A}$ matrix elements:

$$
\begin{aligned}
{[f(A)]_{i j}=} & U_{i k} f\left(d_{k}\right) U_{k j}^{\dagger}=\delta_{i j} f\left(a_{i}^{0}\right)+\left(\frac{f\left(a_{i}^{0}\right)-f\left(a_{j}^{0}\right)}{a_{i}^{0}-a_{j}^{0}}\right) \widetilde{A}_{i j}+ \\
& +\sum_{\ell=1,(\ell \neq i, j)}^{n}\left(\frac{\frac{f\left(a_{i}^{0}\right)-f\left(a_{\ell}^{0}\right)}{a_{i}^{0}-a_{\ell}^{0}}-\frac{f\left(a_{j}^{0}\right)-f\left(a_{\ell}^{0}\right)}{a_{j}^{0}-a_{\ell}^{0}}}{a_{i}^{0}-a_{j}^{0}}\right) \widetilde{A}_{i \ell} \widetilde{A}_{\ell j}+\ldots
\end{aligned}
$$

In case of degenerate eigenvalues, the ill-defined ratios in (3.6) should be replaced by appropriate derivatives. The first line of eq. (3.6) has been presented in ref. [58]. ${ }^{9}$ A formal proof of eq. (3.6) generalised to all orders in powers of $\widetilde{A}$ and its applications to flavour physics will be given elsewhere [59].

For our purpose here, we only require the implementation of eq. (3.6) to the relevant expressions in eq. (3.5). The zero external momentum expansion of self energies and penguins respectively gives (we use here $\hat{I}^{I J}$ as for a Kronecker "delta" symbol, to avoid confusion with other notation for supersymmetric parameters):

$$
\begin{aligned}
\sum_{i=1}^{6} Z_{U}^{J i *} Z_{U}^{I+3, i} B_{0}\left[0, r_{i}\right]=\hat{\Delta}^{J, I+3} C_{0}\left[0 ; r_{J}, r_{I+3}, 1\right] & \\
& +\sum_{K=1}^{6} \hat{\Delta}^{J K} \hat{\Delta}^{K, I+3} D_{0}\left[0 ; r_{J}, r_{K}, r_{I+3}, 1\right] \\
& +\sum_{K, M=1}^{6} \hat{\Delta}^{J K} \hat{\Delta}^{K M} \hat{\Delta}^{M, I+3} E_{0}\left[0 ; r_{J}, r_{K}, r_{M}, r_{I+3}, 1\right]+\mathcal{O}\left(\hat{\Delta}^{4}\right) \\
\sum_{i, l=1}^{6} Z_{U}^{J i *} Z_{U}^{I+3, l} Z_{U}^{K i} Z_{U}^{M l *}\left\{C_{0}, C_{11}\right\}\left[0 ; r_{i}, r_{l}, 1\right]=\hat{I}^{J K} \hat{I}^{M, I+3}\left\{C_{0}, C_{11}\right\}\left[0 ; r_{J}, r_{I+3}, 1\right] & \\
& +\hat{I}^{J K} \hat{\Delta}^{M, I+3}\left\{D_{0}, D_{11}\right\}\left[0 ; r_{J}, r_{M}, r_{I+3}, 1\right] \\
& +\hat{I}^{M, I+3} \hat{\Delta}^{J K}\left\{D_{0}, D_{12}\right\}\left[0 ; r_{J}, r_{K}, r_{I+3}, 1\right] \\
& +\hat{\Delta}^{J K} \hat{\Delta}^{M, I+3}\left\{E_{0}, E_{12}\right\}\left[0 ; r_{J}, r_{K}, r_{M}, r_{I+3}, 1\right] \\
& +\hat{I}^{J K} \sum_{N=1}^{6} \hat{\Delta}^{M N} \hat{\Delta}^{N, I+3}\left\{E_{0}, E_{11}\right\}\left[0 ; r_{J}, r_{M}, r_{N}, r_{I+3}, 1\right] \\
& +\hat{I}^{M, I+3} \sum_{N=1}^{6} \hat{\Delta}^{J N} \hat{\Delta}^{N K}\left\{E_{0}, E_{13}\right\}\left[0 ; r_{J}, r_{N}, r_{K}, r_{I+3}, 1\right]+\mathcal{O}\left(\hat{\Delta}^{3}\right)
\end{aligned}
$$

in terms of the diagonal and non-diagonal elements of the dimensionless squark mass matrix

$$
r_{K}^{2} \equiv \frac{\left(\mathcal{M}_{U}^{2}\right)^{K K}}{m_{\tilde{g}}^{2}}, \quad \hat{\Delta}^{K M} \equiv \frac{\left(\mathcal{M}_{U}^{2}\right)^{K M}}{m_{\tilde{g}}^{2}} \quad(K \neq M), \quad \hat{\Delta}^{K K} \equiv 0,
$$

\footnotetext{
${ }^{9}$ We would like to thank A. Romanino for correspondence on this point.
} 
respectively. The quartic product of $Z_{U}$ matrices in eq. (3.7b) appears after substituting the explicit form of the $V_{H U U}$ vertex in (3.5). The "higher derivative" PV functions $D, E$ are defined in $\mathrm{B}$, together with iterative relations generating them from $B$ and $C$ functions.

An important feature of this "flavour expansion" framework, also noted in ref. [43], is that it allows for a common treatment of completely different flavour structures in $\mathcal{M}_{U}^{2}$. It may apply with the same efficiency in the "degenerate" case where the diagonal elements $r_{K}$ are considered to be equal and the mass splitting originates only from $\hat{\Delta}$ or in the "hierarchical" case where the mass splitting from $\hat{\Delta}$ adds to a pre-existing hierarchical pattern in $r_{K}$.

Substituting (3.7) into (3.5) in zero external momentum approximation for $\Delta F_{L}$ and using the explicit form of the vertex $V_{H U U}$ from A, the aforementioned partial cancellations between self-energy and penguin can be seen to take place. While (3.5a) itself has the proper decoupling behaviour, after adding (3.5b) and (3.5c) only few terms survive, remarkably only those with a good decoupling behaviour. After cancellations and in the most general case where the non-holomorphic trilinear couplings $A_{U}^{\prime}$ are also present, the scale dependence of the leading remnants in $C_{L}^{(h) I J}$, will behave as

$$
\begin{aligned}
(3.5 \mathrm{~b}): & \sim A_{U}^{J I} \frac{\cos (\alpha-\beta)}{\sin \beta} \times \mathcal{O}\left(\frac{1}{M_{S}}\right) \\
& \sim\left(\mu^{\star} Y^{J}+A_{U}^{\prime J J}\right) \delta_{R R}^{J I} \frac{\cos (\alpha-\beta)}{\sin \beta} \times \mathcal{O}\left(\frac{1}{M_{S}}\right) \\
& \sim \delta_{L R}^{J I}\left(\frac{\cos \alpha}{\sin \beta}\right) \times \mathcal{O}\left(\frac{m_{t}}{M_{S}}\right) \\
& \sim \delta_{L R}^{J J} \delta_{R R}^{J I}\left(\frac{\cos \alpha}{\sin \beta}\right) \times \mathcal{O}\left(\frac{m_{t}}{M_{S}}\right) \\
& \sim \sum_{A, B=1}^{3} \delta_{L R}^{J A} \delta_{R L}^{A B} \delta_{L R}^{B I}\left(\frac{\cos \alpha}{\sin \beta}\right) \times \mathcal{O}\left(\frac{M_{S}}{m_{t}}\right), \\
(3.5 \mathrm{a}): & \sim \delta_{R R}^{J I}\left(\frac{\cos \alpha}{\sin \beta}\right) \times \mathcal{O}\left(\frac{m_{t}^{2}}{M_{S}^{2}}\right) \\
& \sim \sum_{A=1}^{3} \delta_{R L}^{J A} \delta_{L R}^{A I}\left(\frac{\cos \alpha}{\sin \beta}\right) \times \mathcal{O}(1)
\end{aligned}
$$

where we have expressed our results in terms of the more useful $3 \times 3$ block matrices $\delta$. These are defined through,

$$
\hat{\Delta} \equiv\left(\begin{array}{ll}
\delta_{L L} & \delta_{L R} \\
\delta_{R L} & \delta_{R R}
\end{array}\right): \delta_{L R}=\left(\delta_{R L}\right)^{\dagger}, \delta_{L L}^{A A}=\delta_{R R}^{A A}=0, \quad(A=1, \ldots 3)
$$

The analytic expressions in (3.9) reveal certain regions in MSSM parameter space where $\mathcal{B}(t \rightarrow q h)$ is enhanced and could be accessible in the high luminosity LHC data. We will investigate these enhanced scenarios in section 5 . 
The scaling behaviour of the leading contributions presented in (3.9) is obtained after considering all SUSY mass parameters scaling simultaneously as $\sim M_{S}$ and all electroweak mass parameters as $\sim m_{t}$ in the full expression for the leading remnants using (3.6). Under these assumptions the blocks of $\hat{\Delta}$ in eq. (3.10) will behave as

$$
\delta_{L L} \sim \delta_{R R} \sim \mathcal{O}(1), \delta_{L R} \sim \mathcal{O}\left(\frac{m_{t}}{M_{S}}\right)
$$

due to the non-uniform scaling of the respective blocks inside $\mathcal{M}_{U}^{2}$. Using this observation, it is important to notice that all leading remnants in (3.9) scale as $\mathcal{O}\left(m_{t}^{2} / M_{S}^{2}\right)$, which is straightforward to see for all contributions besides the $\sim \cos (\alpha-\beta)$ terms arising from (3.5b). These at first sight seem to exhibit a non-decoupling behaviour, however, a closer look reveals that the decoupling is hidden within the quantity

$$
\frac{\cos (\alpha-\beta)}{\sin \beta} \stackrel{\text { SM-limit }}{\simeq} 2 \cos \beta \cos (2 \beta) \frac{M_{Z}^{2}}{M_{A}^{2}} \sim \mathcal{O}\left(\frac{m_{t}^{2}}{M_{S}^{2}}\right),
$$

and their contribution can become comparable with all other terms, obviously subject to the $\tan \beta$ value chosen.

The flavour structure of (3.9) may provide us with useful guiding information on the leading dependence of $C_{L}^{(h) I J}$ in terms of the Lagrangian parameters involved. For example, for $t \rightarrow c h$-amplitude $\left[J=3, I=2\right.$ in (3.9)], the parameters directly involved are $A_{U}^{\prime 32}, \delta_{R R}^{32}$ and $A_{U}^{32}$ with the last parameter always introduced through the $\delta_{L R}^{32}$ squark mass matrix element. At a secondary level, flavour conserving parameters such as $\mu$ or $\delta_{L R}^{33} \sim A_{t}$ may enter the expressions, however only as pre-factors of the previous ones. As a result they modify substantially the final result of $\mathcal{B}(t \rightarrow c h)$. Analogous results hold for $\mathcal{B}(t \rightarrow u h)$, with obvious superscript replacements $2 \rightarrow 1$ into parameters above.

At this point, it seems instructive to present numerically, in figure 2, the cancellation of self energy and penguin contributions in $C_{L}^{(h) I J}$ for a typical choice of the parameters involved, and for uniform scaling case $M_{A}=m_{\tilde{g}}=M_{S}$ (for the examples illustrated in figure 2 we ignore experimental bounds on $\delta$-parameters). We choose to present results in $t \rightarrow c h$ amplitude but analogous results hold also for the $t \rightarrow u h$ amplitude. It is clear from figure 2 (left), where we plot the full numerical result for the Wilson coefficient $\left|C_{L}^{(h) 23}\right|$ with respect to $M_{S}$, that the non-decoupling behaviour of the penguin $\left(\Delta F_{L}\right)$ cancels the non-decoupling behaviour of the self-energy diagrams leaving behind remnants in $\left|C_{L}^{(h) 23}\right|$ which are decreasing as $m_{t}^{2} / M_{S}^{2}$. This is scaling behaviour exactly as our approximate expressions in (3.9) indicate.

An analogous situation is realised in figure 2(right) in the case of $\left|C_{R}^{(h) 23}\right|$ for which, due to the aforementioned $L \leftrightarrow R$ symmetry in the expression for the Wilson coefficients in (3.9), the result primarily depends on $\delta_{L L}^{32}, A_{U}^{\prime 23 *}$ and $A_{U}^{23 *}$. This clear decoupling behaviour is in qualitative agreement with ref. [20], for $M_{A}=M_{S}$.

One should note that the terms listed in (3.9) are leading or next to leading order contributions in terms of $\delta$-parameters, obtained in the approximation of vanishing momenta of the external particles. Under the uniform scaling of all SUSY parameters these terms scale as $\sim m_{t}^{2} / M_{S}^{2}$. There are other contributions that scale similarly and can be 

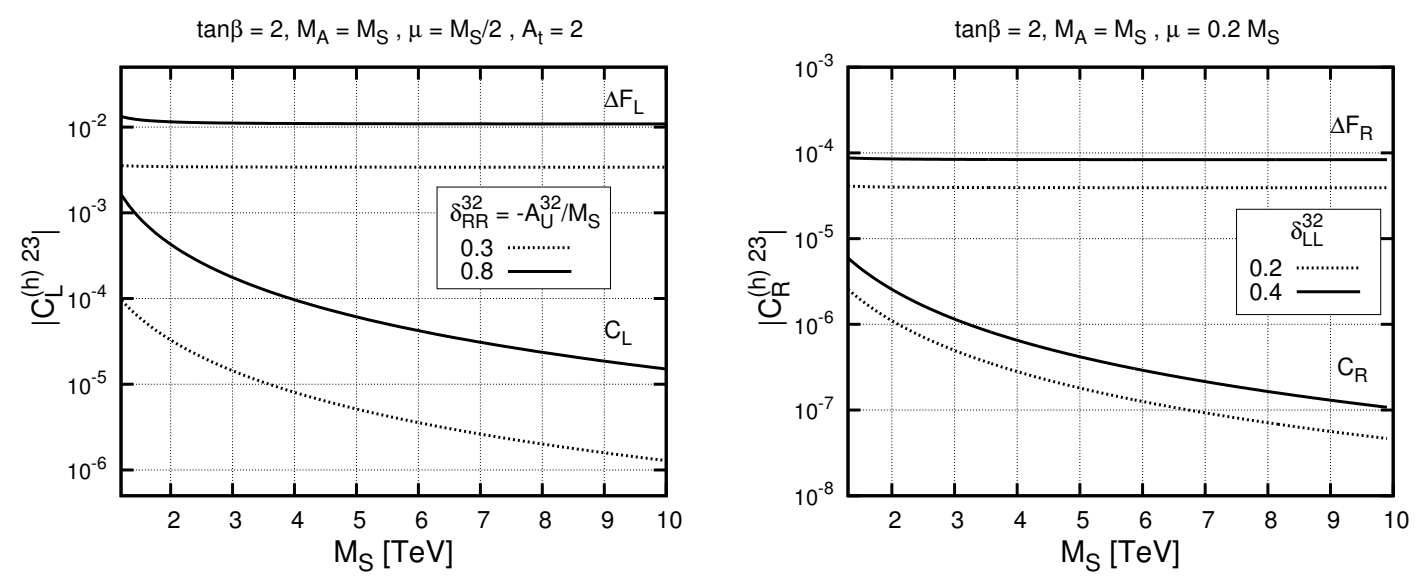

Figure 2. Left: cancellation and remnants of $\left|C_{L}^{(h) 23}\right|$ for two values of the non-diagonal squark mass parameter $\delta_{R R}^{32}$, assumed here to be related to trilinear term as $\delta_{R R}^{32}=-A_{U}^{32} / M_{S}$, in the case of degenerate squark mass spectrum $\left(r_{K}^{2}=1\right)$ and a uniform scaling $\left(M_{A}=m_{\tilde{g}}=M_{S}\right)$. The penguin contribution (upper lines) is denoted by $\left(\Delta F_{L}\right)$. Right: Similarly, for the Wilson coefficient $\left|C_{R}^{(h) 23}\right|$ and for two values of $\delta_{L L}^{32}$ parameter.

extracted from the full amplitude expression (3.5). For example, the first non-trivial order in the external momentum expansion of the penguin amplitude $\delta F_{L(R)}\left(k_{1}, k_{2}\right)$ has the similar flavour structure and decoupling properties, so it will modify the coefficients of terms in (3.9) but does not change our qualitative discussion. Other possible terms, e.g. higher order contributions in the flavour expansion, are either subleading in $\delta$ 's or small due to other suppression factors, so we do not display them explicitly. They are of course included in the numerical analysis presented in next sections, as for that we use full unexpanded formulae (2.10) and (2.13).

Finally, similar cancellations of non-decoupling contributions can be observed numerically (and as we checked also analytically, although after more complicated calculations) for chargino and neutralino contributions to the considered $t \rightarrow q h$ decay amplitude. Therefore, they always become smaller than the gluino diagrams, independently of the soft SUSY breaking parameters scale (see also footnote 3 ).

\section{Constraints from other observables}

As we discussed already in section 2 we have added our calculations for $\mathcal{B}(t \rightarrow q h)$ into the SUSY_FLAVOUR library [44-46]. For every input MSSM parameter set, SUSY_FLAVOUR calculates a number of $B-, K$-, and $D$-meson physics observables. Comparing them with experimental bounds [60] allows us to plot predictions for the $t \rightarrow q h$ decay rate only for realistic values of the MSSM parameters.

Most of these observables are related to the processes involving down quarks and they constrain strongly the flavour structure of $m_{Q_{L}}^{2}$ soft mass matrix, common from both $\tilde{D}$ and $\tilde{U}$ squarks. Thus, it is unlikely to have $\delta_{L L}^{I 3} \gtrsim 10 \%$ and this is impossible to generate large effects in $t \rightarrow q h$ decays. We are therefore going to set $\delta_{L L}^{i 3}$ zero in the numerical results 


\begin{tabular}{|cc|}
\hline Quantity & Current Measurement \\
\hline$m_{\tilde{g}}$ & $>1.1 \mathrm{TeV}$ \\
single light squark $m_{\tilde{q}}$ & $>500 \mathrm{GeV}$ \\
$m_{\tilde{t}_{L}}$ & $>600 \mathrm{GeV}$ \\
$m_{\tilde{t}_{R}}$ & $>200 \mathrm{GeV}$ \\
$m_{h}$ & $(125.9 \pm 0.4) \mathrm{GeV}$ \\
\hline Neutron EDM $\left(\left|d_{n}\right|\right)$ & $<2.9 \cdot 10^{-26} \mathrm{e} \mathrm{cm}[64]$ \\
\hline
\end{tabular}

Table 1. Experimental bounds used throughout in our numerical analysis.

below. For $\delta_{L R}^{I 3}$ and $\delta_{R R}^{I 3}$ and at low and moderate values of $\tan \beta$, potentially important constraints for $\mathcal{B}(t \rightarrow q h)$ arise from the D-meson mass difference, $\Delta M_{D}$. However, $\Delta M_{D}$ is particularly sensitive to $\delta_{R R}^{12}$ element, which affects $\mathcal{B}(t \rightarrow q h)$ only through higher powers of $\delta$-insertions than those attributed to the leading effect in (3.9). Also $\mathcal{B}\left(B \rightarrow X_{s} \gamma\right)$ and $\bar{B}_{s(d)}-B_{s(d)}$ mixing could be potentially bound to constraints but they are not significant as contributions from the right up-squark sector to these processes are suppressed by the powers of light quark Yukawa couplings.

There are of course relevant constraints for parameters important for $\mathcal{B}(t \rightarrow q h)$ emerging from direct, mainly LHC, SUSY searches [60,61]. These are shown in table 1 . A scenario which is particularly interesting for enhancing $\mathcal{B}(t \rightarrow q h)$ is the one with the light stop mostly "right handed". In this case a lower bound for light stop, together with a nearly degenerate neutralino, as low as $m_{\tilde{t}_{R}} \approx 200-400 \mathrm{GeV}$ cannot be excluded in current LHC data [61-63].

The recent discovery of the Higgs boson mass at LHC [7, 8], if interpreted as a "natural" MSSM light Higgs boson, requires a large, often close to maximal, trilinear soft breaking coupling $\delta_{L R}^{33} \propto A_{t} / M_{S} \approx \sqrt{6}$. In fact, this helps $\mathcal{B}(t \rightarrow q h)$ to be enhanced as we observe from our qualitative results in (3.9). We have incorporated in SUSY_FLAVOUR two-loop approximate expressions for the CP-even Higgs bosons, based on ref. [65] for contributions from the top/stop sector, and supplied with results from ref. [66] for contributions from other sectors. As stated in ref. [65], such approximation should reproduce the full 2-loop result for the Higgs boson mass with accuracy better than $2 \mathrm{GeV}$. Therefore, we allow for a region $123 \mathrm{GeV} \lesssim m_{h} \lesssim 128 \mathrm{GeV}$, because of unaccounted theory errors from higher loop corrections. Note that full 2-loop formula for the MSSM CP-even Higgs boson mass has not been calculated yet in the fully general flavour violating case, with large off-diagonal squark mass insertion. Thus, actual theoretical error of expressions given in ref. [65] can be bigger, affecting the Higgs mass constraints.

\section{Results}

Our goal here is to find out the maximal outcome on $\mathcal{B}(t \rightarrow q h)$ in the MSSM. By reading (3.9) the maximal effect on $\mathcal{B}(t \rightarrow q h)$ will be led by the following parameters [FC 


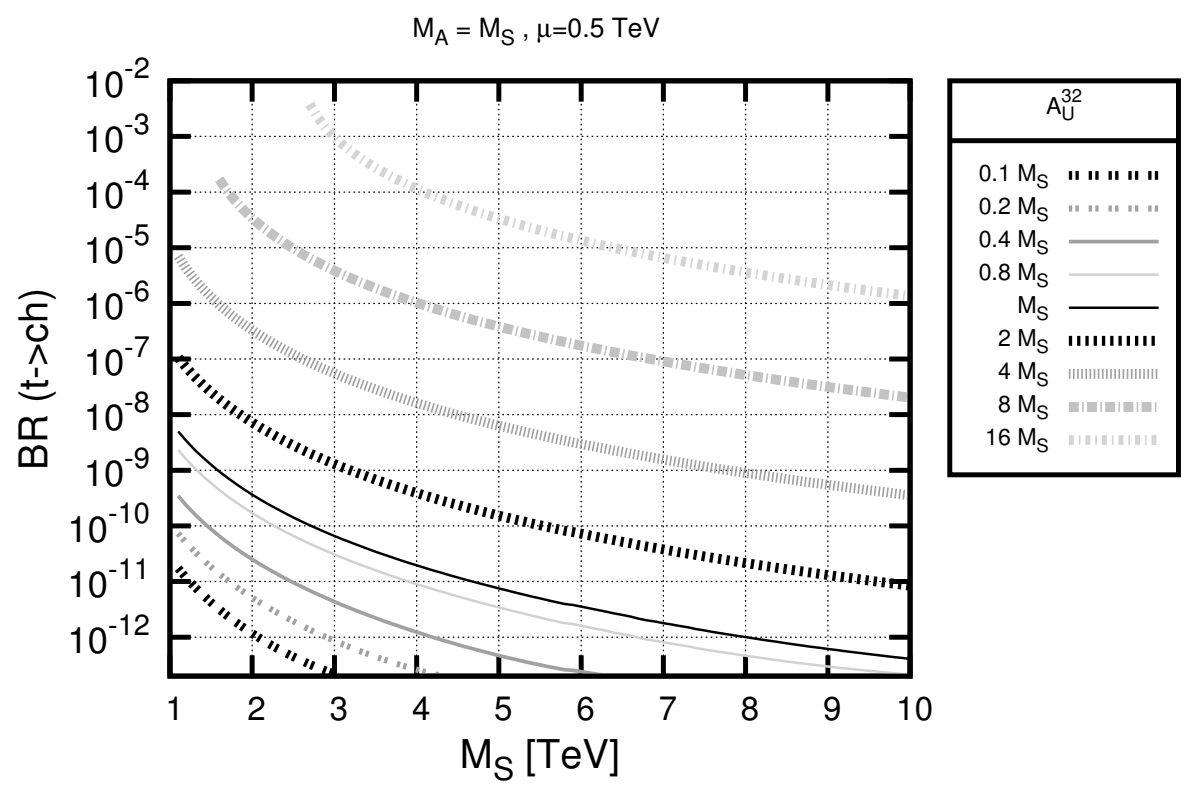

Figure 3. Enhancing the $t \rightarrow c h$ decay rate by varying the $A_{U}^{32}$ parameter for a degenerate spectrum $\left(r_{K}=1\right)$ and a uniform scaling $\left(m_{\tilde{g}}=M_{A}=M_{S}\right) . A_{t} \approx 2 M_{S}$ and $2 \lesssim \tan \beta \lesssim 4$ are assumed to be consistent with the measured Higgs boson mass of table 1. The position of the left edge of each line corresponds to the condition $m_{\tilde{t}_{L}} \geq 600 \mathrm{GeV}$.

stands for Flavor Changing and $\left.A_{t}\left(A_{t}^{\prime}\right) \equiv A_{U}^{33}\left(A_{U}^{\prime 33}\right)\right]$ :

$$
\begin{aligned}
& \text { Non - FC : } A_{t}, \quad A_{t}^{\prime}, \quad m_{\tilde{t}_{L}}, \quad m_{\tilde{t}_{R}}, \quad \mu, \quad m_{\tilde{g}}, \tan \beta, \quad M_{A}, \\
& \text { Holomorphic (FC) : } \quad \delta_{L L}^{I 3}, \quad \delta_{R R}^{I 3}, \quad A_{U}^{I 3}, \quad A_{U}^{3 I} \text {, } \\
& \text { Non - Holomorphic (FC) : } A_{U}^{\prime I 3}, \quad A_{U}^{\prime 3 I} .
\end{aligned}
$$

Below we present full numerical results mostly for $\mathcal{B}(t \rightarrow c h)$. This is affected by $(I=2)$ parameters in eqs. (5.2) and (5.3). Results for $\mathcal{B}(t \rightarrow u h)$ are exactly the same as one can see from the leading order expansion (3.9) with the obvious replacement $(I=1)$ in the parameters of eqs. (5.2) and (5.3). Constraints from neutron EDMs are stronger on the latter and as a result we consider mainly $\mathcal{B}(t \rightarrow c h)$ in investigating observability at LHC.

As we have remarked earlier, the analytic formulae, eq. (3.9), allow for the occurrence of enhanced effects in certain regions of parameter space. This saves us from time consuming, and often difficult to understand and interpret, grid-scan plots. Consequently, the following possibilities for an enhanced $\mathcal{B}(t \rightarrow q h)$ emerge.

\subsection{Enhancement through large $\frac{\left|A_{U}^{3 I}\right|}{M_{S}}$ and $\frac{\left|A_{U}^{I 3}\right|}{M_{S}}$}

Inspection of (3.9) shows that in leading approximation the expression for $\mathcal{B}(t \rightarrow q h)$ contains several terms depending on up-squark trilinear mixing parameters $A_{U}^{I J}$. The 
relevant terms in $C_{L}^{(h)}$ (for $C_{R}^{(h)}$ one needs to exchange chiral indices $L \leftrightarrow R$ ) are

$$
\begin{aligned}
C_{L}^{(h)}: & \sim \delta_{L R}^{J I}\left(\frac{\cos \alpha}{\sin \beta}\right) \mathcal{O}\left(\frac{m_{t}}{M_{S}}\right), \quad \sim \sum_{A, B=1}^{3} \delta_{L R}^{J A} \delta_{R L}^{A B} \delta_{L R}^{B I}\left(\frac{\cos \alpha}{\sin \beta}\right) \mathcal{O}\left(\frac{M_{S}}{m_{t}}\right) \\
& \sim \sum_{A=1}^{3} \delta_{R L}^{J A} \delta_{L R}^{A I}\left(\frac{\cos \alpha}{\sin \beta}\right) \mathcal{O}(1) .
\end{aligned}
$$

Thus, large $A_{U}^{I J}$ values can enhance the discussed decay rates.

Such scenario is illustrated in figure 3 , where we plot $\mathcal{B}(t \rightarrow c h$ ) (so that $J=3$ and $I=2$ ) as a function of $M_{S}=m_{\tilde{g}}=M_{A}$ for various values of $A_{U}^{32} / M_{S}$ and for a fixed value of $A_{t}=2 M_{S}$. In addition, the higgsino mass parameter is set to $\mu=0.5 \mathrm{TeV}$ and all other non-diagonal elements of $\delta$ vanish. For simplicity in figure 3 we vary only $A_{U}^{32} / M_{S}$, setting it to several real-positive values, however as can be seen from analytic formulae, the result for $\mathcal{B}(t \rightarrow c h)$ is symmetric under replacement $A_{U}^{32} \leftrightarrow A_{U}^{23}$ and depends primarily on the absolute values of both parameters, so we do not discuss dependence on $A_{U}^{23}$ separately.

As can be seen from (5.4), the form factor $C_{L}^{(h)}$ contain terms with linear dependence and a term with cubic dependence in $\delta_{L R}^{32}=-\frac{v_{2} A_{U}^{32}}{\sqrt{2} M_{S}^{2}}$. Linear dependence dominates for $A_{U}^{32} / M_{S} \ll 1$ while, more importantly, cubic dependence dominates for $A_{U}^{32} / M_{S} \gg 1$. As it is obvious from second line of (5.4), the parameter $A_{t} \approx 2 M_{S}$, required for a $126 \mathrm{GeV}$ Higgs boson mass, enhances $\left|C_{L}\right|$ and therefore $\mathcal{B}(t \rightarrow c h)$, only in parameter regions where linear dependence dominates, namely for $A_{U}^{32} / M_{S} \ll 1$. In the more interesting cubic dependence region, where $A_{U}^{32} / M_{S} \gg 1$ and the maximal values of $\mathcal{B}(t \rightarrow c h)$ are obtained, the branching ratio can reach LHC attainable values, exceeding estimate (1.7) by two orders of magnitude, for $A_{U}^{32} \gtrsim 8 M_{S}$ and for a light $M_{S}$ value, as can be seen in the left upper corner of figure 3 . There, the minimum value of $M_{S}$ is subject to the condition that the left handed stop squark mass is heavier than $600 \mathrm{GeV}$, as table 1 indicates.

We should note that our results for $\mathcal{B}(t \rightarrow c h)$ shown in figure 3 do not display any non-decoupling effect. The decay rate increases with the $A_{U}^{32} / M_{S}$ ratio, but for each fixed choice of $A_{U}^{32} / M_{S}$ it decreases as our analytic formulae indicate, i.e., as $\left|C_{L}\right|^{2} \sim m_{t}^{4} / M_{S}^{4}$. In the most interesting region $A_{U}^{32} / M_{S} \gg 1$, where the cubic dependence in $\delta_{L R}^{32}$ dominates $C_{L}$, the branching ratio behaves as

$$
\mathcal{B}(t \rightarrow c h) \propto\left(\frac{A_{U}^{32}}{M_{S}}\right)^{6} \mathcal{O}\left(\frac{m_{t}^{4}}{M_{S}^{4}}\right) .
$$

A small deviation from this behaviour can be seen on the left edge of the upper curves where steeper slopes appear due to $\left|\delta_{L R}^{32}\right|$ closing to unity and higher order corrections becoming increasingly important. For large $M_{S}$, deep in the SM (decoupling)-limit, although the effect is substantially smaller, the $\mathcal{B}(t \rightarrow c h)$ is still enhanced by many orders of magnitude as compared to the SM prediction.

Another important remark should be done concerning how realistic are very large values of $\left|A_{U}^{32}\right| / M_{S}$ (or $\left.\left|A_{U}^{23}\right| / M_{S}\right)$, required to enhance the $\mathcal{B}(t \rightarrow c h$ ). As previously mentioned, they are always constrained by the condition $\left|\delta_{L R}^{32(23)}\right| \lesssim 1$ resulting from the 
light stop mass bound:

$$
\left|\delta_{L R}^{32}\right| \sim \frac{v_{2}}{\sqrt{2} M_{S}} \frac{\left|A_{U}^{32}\right|}{M_{S}} \lesssim 1 \quad \longrightarrow \quad \frac{\left|A_{U}^{32}\right|}{M_{S}} \lesssim \frac{\sqrt{2} M_{S}}{v_{2}} .
$$

Thus, in principle even very large values of $\left|A_{U}^{32}\right| / M_{S}$ are possible assuming sufficiently high SUSY mass scale, e.g. for $M_{S}>1.5 \mathrm{TeV}$ one can reasonably consider $A_{U}^{32} \sim 8 M_{S}$. However, such large $A_{U}$ in connection with light stop mass square can possibly trigger unwanted Charge and Colour Breaking minima (CCB) [67-78]. For example, allowing for non-vanishing $A_{U}^{32}$ and following the steps of ref. [69], and assuming possible vevs in the five dimensional field space direction, $H_{1}^{0}=0, H_{2}^{0}=1, \tilde{t}_{L}=0, \tilde{t}_{R}=\tilde{c}_{R}=1,{ }^{10}$ we arrive analytically at the following constraint,

$$
\left|A_{U}^{32}\right|^{2} \leq Y_{t}^{2}\left(m_{H_{2}}^{2}+m_{\tilde{t}_{L}}^{2}+m_{\tilde{c}_{R}}^{2}+\mu^{2}\right),
$$

in agreement with ref. [73]. One can arrive at an even stronger bound involving both $\left|A_{t}\right|$ and $\left|A_{U}^{32}\right|$, which is appreciable because of the Higgs mass constraint, following the field direction $\tilde{t}_{L}=H_{2}^{0}=1, H_{1}^{0}=0, \tilde{t}_{R}=\tilde{c}_{R}=1 / \sqrt{2}$,

$$
\left(\left|A_{t}\right|+\left|A_{U}^{32}\right|\right)^{2} \leq 4 Y_{t}^{2}\left[m_{H_{2}}^{2}+m_{\tilde{t}_{L}}^{2}+\frac{1}{2}\left(m_{\tilde{t}_{R}}^{2}+m_{\tilde{c}_{R}}^{2}\right)\right]^{2},
$$

in agreement with a similar one found recently in ref. [79]. For a common squark and Higgs mass scale, $M_{S}$, the constraint (5.7) results in $\left|A_{U}^{32}\right| \lesssim \sqrt{3} M_{S}$, which is far more stringent than the positivity physical mass squared constraint of (5.6). For such values of $A_{U}^{32}$, and, after reading from figure 3 , we deduce that

$$
\mathcal{B}(t \rightarrow c h) \lesssim 10^{-7}
$$

This rate is out of any near future LHC expected sensitivity [see (1.4)].

A detailed analysis of the CCB problem in the general flavour violating MSSM is beyond the scope of this paper. Nevertheless, in most cases the issue is a cosmological one, since sometimes the inverse transition rate between meta-stable vacua exceeds the lifetime of the universe. In this case, the pre-factor of $\mathcal{O}(1)$ in the r.h.s. of eq. (5.7) may be modified, but it is unlikely that it increases by an order of magnitude or so, necessary to achieve $\mathcal{B}(t \rightarrow c h) \sim 10^{-4}$. This claim is supported by the results of ref. [75], where the bound in eq. (5.7) is only marginally relaxed by meta-stability. For recent accounts on meta-stability of the MSSM vacuum in MFV scenario, see refs. [76-78]. ${ }^{11}$

In a more general case both $A_{U}^{32}$ and $\delta_{R R}^{32}$ parameters can be present simultaneously. In this case the possibly largest contributions to the $C_{L}^{(h)}$ form factor, out of all listed in (3.9), are given by terms

$$
\sim \delta_{L R}^{J J} \delta_{R R}^{J I}\left(\frac{\cos \alpha}{\sin \beta}\right) \times \mathcal{O}\left(\frac{m_{t}}{M_{S}}\right), \quad \sim \delta_{L R}^{J I} \delta_{R L}^{I J} \delta_{L R}^{J I}\left(\frac{\cos \alpha}{\sin \beta}\right) \times \mathcal{O}\left(\frac{M_{S}}{m_{t}}\right) .
$$

\footnotetext{
${ }^{10}$ Fields are normalized to $H_{2}^{0}$ and we take the limit, $Y_{c}^{2} / Y_{t}^{2} \rightarrow 0$.

${ }^{11} \mathrm{~A}$ more robust check for CCB vacua can be studied with the publicly available code Vevacious [80] which performs a full numerical check of the potential (meta)stability even at 1-loop level. A thorough scan of the interesting parameter space can be however limited by long computer run-time.
} 


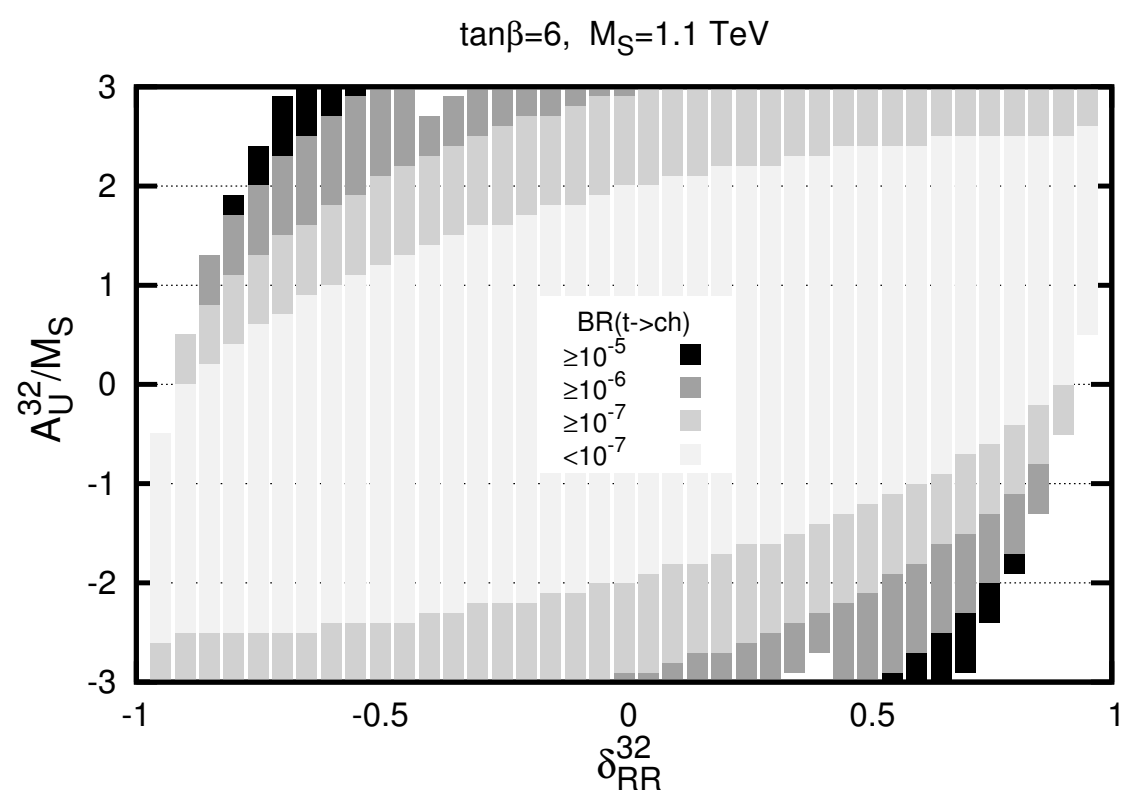

Figure 4. Contour plot for $\mathcal{B}(t \rightarrow c h)$ prediction on a $\delta_{R R}^{32}$ vs. $A_{U}^{32} / M_{S}$ plane. All other FC parameters are set to zero. In addition, we take $\left(m_{\tilde{g}}=M_{A}=\mu=M_{S}\right)$ and $A_{t} / M_{S}=2$ while other values are shown in the figure.

In figure 4 we plot $\mathcal{B}(t \rightarrow c h)$ on the $\delta_{R R}^{32}$ and $A_{U}^{32} / M_{S}$ plane, varying $A_{U}^{32}$ within the region $\left|A_{U}^{32}\right| / M_{S} \lesssim 3$ in order to avoid potential CCB bounds. Note that contributions from these two parameters can interfere constructively (top-left and bottom-right corners of the plot) or destructively (bottom-left and top-right corners). However, even in the most optimistic case, the branching ratio $\mathcal{B}(t \rightarrow c h)$ cannot exceed values of order $\sim 10^{-5}$ which is an order of magnitude less than the expected sensitivity of LHC.

An analogous effect for $\mathcal{B}(t \rightarrow c h)$ may also arise from the $C_{R}^{(h)}$ contribution, namely from the $A_{U}^{23 *}$ and $\delta_{L L}^{32}$ pair of parameters. However including such an effect has little to offer since the enhancement that could be obtained this way (factor 2 at most) is suppressed due to the stringent experimental bounds on $\delta_{L L}$.

In figure 5 we present results for $\mathcal{B}(t \rightarrow u h)$ on a $\delta_{R R}^{31}$ vs. $A_{U}^{31} / M_{S}$ plane. As we have already discussed, formulae for this decay are exactly the same as for $\mathcal{B}(t \rightarrow c h)$, with obvious replacements of indices of flavour violating parameters. However, the important difference comes from the fact that $A_{U}^{31}, A_{U}^{13}$ and $\delta_{R R}^{13}$ are highly constrained by experimental bound on neutron Electric Dipole Moment (EDM), see e.g. [81]. Although we have assumed real parameters throughout this article, this is an effect that arises from the terms of the higher order in the mass insertion expansion of the gluino contribution to the down quark electric and chromoelectric dipole moments. Such terms are proportional to $A_{U}^{31(13)}$ or $\delta_{R R}^{13}$ multiplied by the CKM matrix elements containing imaginary phase. Effects of this kind are usually quite small and unobservable, comparing to experimental and theoretical accuracy with which most of the rare processes is known. However, the bound on neutron EDM is so strong, that it has visible impact on the acceptable ranges of the real soft parameters. Approximately, the whole effect results in a strong correlation of the allowed values of 


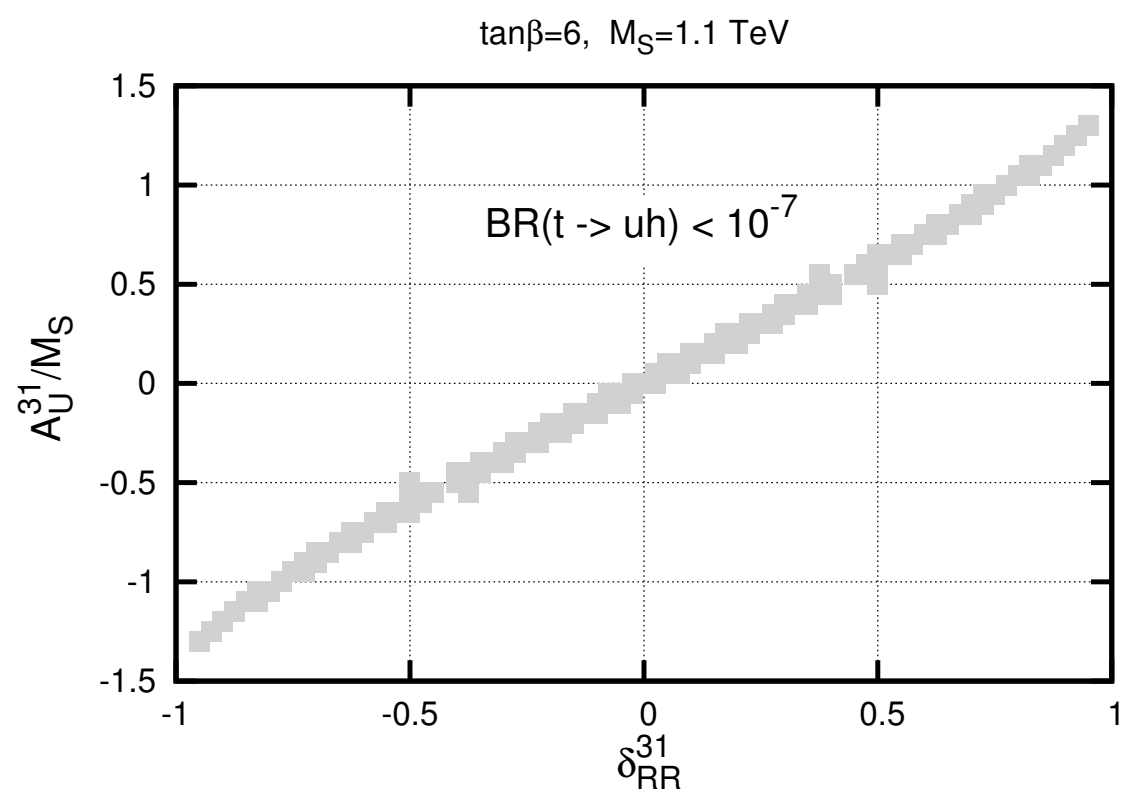

Figure 5. Contour plot for $\mathcal{B}(t \rightarrow u h)$ on a $\delta_{R R}^{31}$ vs. $A_{U}^{31} / M_{S}$ plane with all other parameters set as in figure 4. Due to severe neutron EDM constraints, the effect is confined to a region where the decay rate is far beyond the reach of LHC.

$A_{U}^{31} / M_{S}$ and $\delta_{R R}^{31}$, such that their linear combination with $\mathcal{O}(1)$ coefficients (depending on up-squark and gluino masses) must vanish with $\mathcal{O}\left(10^{-2}\right)$ accuracy, to satisfy the current experimental neutron EDM bound in table 1. As it is obvious from figure 5, we then find $\mathcal{B}(t \rightarrow u h) \lesssim 10^{-7}$ which is unobservable at LHC.

Based on (3.9), one can in principle search how to enhance $\mathcal{B}(t \rightarrow q h)$ other than by previously analysed its cubic dependence on $A_{U}^{32} / M_{S}$. An analogous effect may also be produced by increasing $\sim \delta_{R R}^{32}$ together with the unnatural choice of $|\mu| / M_{S} \gg 1$. Even so, such a contribution is suppressed by the condition $\delta_{R R}^{32}<1$ and thus will be typically subleading, unless $\mu / M_{S} \gg A_{U}^{32} / M_{S}$. Therefore, the parameter space exploited in figures 3 and 4 seems to be the optimal one.

Finally, for comparison with the recent literature, we recalculate results presented in Scan-I of ref. [20]. We find numerical agreement for $\mathcal{B}(t \rightarrow c h)$ within $10 \%$. This may be understandable since we take into account QCD renormalization group running effects and threshold corrections for Wilson-coefficients neglected in ref. [20] or in other literature quoted in the introduction section.

\subsection{The light $M_{A}$ scenario and non-holomorphic dominance}

The second enhancement scenario requires a light Higgs sector and significant contribution from the non-holomorphic trilinear soft couplings, $A_{U}^{\prime}$. The numerical results are displayed in figure 6 . We shall attempt here an explanation of the enhancement based on analytic expansion in (3.9). We must warn the reader however that this case scenario is disfavoured by LHC data and we mostly present it here for complementarity reasons. 

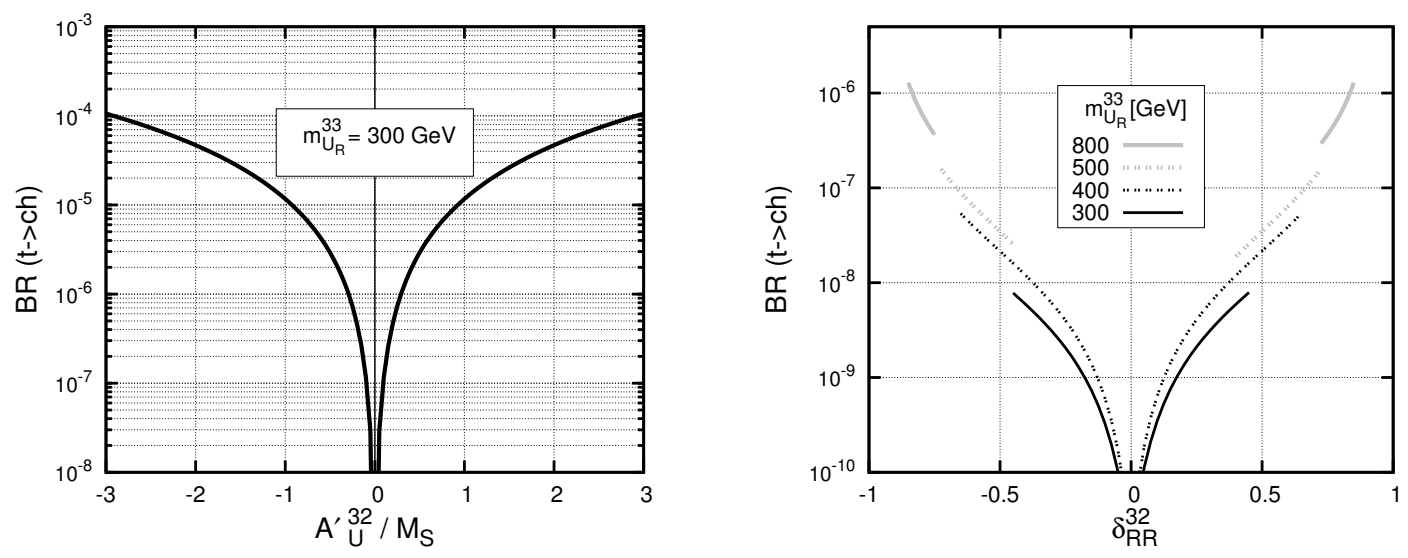

Figure 6. Branching ratios for the light $M_{A}=110 \mathrm{GeV}$ scenario and chosen set of MSSM parameters: $\tan \beta=6, \mu=250 \mathrm{GeV}, M_{S}=1.1 \mathrm{TeV}, A_{t} / M_{S}=2.7$. The leading contribution (left panel) originates from the non-holomorphic coupling $A_{U}^{\prime 32}$. If $A_{U}^{\prime 32} \approx 0$ the next to leading contribution (right panel) is controlled by $\left(\mu^{*} \delta_{R R}^{32}\right)$. The allowed $\delta_{R R}^{32}$ range for each $m_{U_{R}}^{33}$ value corresponds to the $200<m_{\tilde{t}_{R}}<400 \mathrm{GeV}$ constraint of the "light stop window" [62].

This scenario departs from the assumption of uniform scaling for $M_{A}$ and assume light $M_{A} \sim M_{Z}$. Only terms proportional to $\cos (\alpha-\beta)$ in $(3.5 \mathrm{a}),(3.5 \mathrm{~b})$ will be enhanced [see also eq. (3.12)]. To illustrate the size of possible light $M_{A}$ effects we assume for simplicity vanishing non diagonal squark mass matrix elements beside $A_{U}^{\prime 23}$ and $\delta_{R R}^{32}$ in left and right panels of figure 6 , respectively. In order to make this point quantitative, we follow the scenario of ref. [57] in which the heavy Higgs boson is the one seen at LHC with mass around $125.5 \mathrm{GeV}$ and the light one lies in the region $95 \lesssim m_{h} \lesssim 101 \mathrm{GeV}$ where LEP had seen some small excess in Higgs data.

As we observe from the left panel of figure 6, the non-holomorphic soft breaking term $A_{U}^{\prime 23} \approx 3 M_{S}$ may easily bring $\mathcal{B}(t \rightarrow c h)$ to the level observable in future LHC measurements. This is not true in the right panel of figure 6 , where $\delta_{R R}^{32}$ is varied instead. Here effect is much smaller due to the constraints $\delta_{R R}^{32}<1$ (physical squark masses) and $|\mu|<400 \mathrm{GeV}$ $(b \rightarrow s \gamma)$. In that case we obtain $\mathcal{B}(t \rightarrow c h) \lesssim 10^{-6}$, far off LHC's future sensitivity.

Also the more promising scenario with enhanced non-holomorphic contribution in figure 6 (left panel) can only be realised in particular parameter choice. In such a scenario, the $B \rightarrow X_{s} \gamma$ constraint imposes $\mu \lesssim 400 \mathrm{GeV}$. One of the two charginos, namely the higgsino-like one, with mass proportional to $\mu$, should be light and cancel the charged Higgs terms in the respective penguin diagrams. Thus we choose $\mu$ small and heavy winos in order to split the chargino masses. We have also taken a tuned value for trilinear SUSY breaking coupling, $A_{t} / M_{S}=2.7 \pm 0.05$, which allows to pass the constraints of table 1 and $B \rightarrow X_{s} \gamma$, for a large region in $\mu$, namely $150<|\mu|<350 \mathrm{GeV}$. We could relax the tuning here but only at the cost of severely restricting the $\mu$ parameter space, $\mu \simeq(125 \sim 150)$. In any case $\left|A_{t} / M_{S}\right| \simeq(2 \sim 3)$ is always required in this scenario. Finally, following ref. [57] we can only vary $\tan \beta$ within the $\tan \beta \simeq 6 \sim 7$ region.

In the light of recent searches for charged Higgs boson produced in $t \rightarrow H^{+} b$ decays and decaying to $\tau$ 's [56] this scenario seems increasingly unlikely, at least assuming MSSM 
relations between the Higgs boson masses. In principle, there is an open window (at $1 \sigma$ ) around $6 \lesssim \tan \beta \lesssim 10$ but only at very low $m_{H^{ \pm}}$masses less than $110 \mathrm{GeV}$. Using the MSSM Higgs boson mass sum rule $m_{H^{ \pm}}^{2}=m_{A}^{2}+m_{W}^{2}$, this would require very light $M_{A} \lesssim 75 \mathrm{GeV}$ far below $M_{A}=110 \mathrm{GeV}$ suggested by the LEP possible excess.

\section{Conclusions}

In the present article we have studied rare, flavour-changing top quark decays to light up-quarks $u$ or $c$ and the Higgs boson $h$,

$$
t \rightarrow u h \quad \text { or } t \rightarrow c h
$$

in the framework of MSSM with $R$-parity conservation. Although the corresponding processes in the framework of the Standard Model are highly suppressed, mostly due to the GIM mechanism, such a suppression is not a priori expected in the case of MSSM.

We improve upon existed calculations, most notably from refs. [16-18], by including next to leading order QCD corrections and RGE running from the SUSY soft breaking masses down to $m_{t}$. SUSY finite threshold effects into $t \rightarrow q g$ that mixes with $t \rightarrow q h$, are fully included. This set of most up-to-date one-loop corrections to $t \rightarrow q h$ amplitudes are then included in publicly available SUSY_FLAVOUR library, and therefore combined with MSSM predictions from numerous other flavour physics observables. In addition to current literature, we study effects arising from the non-holomorphic soft SUSY breaking terms. These turn out to be important for enhancing $\mathcal{B}(t \rightarrow q h)$ but in a parameter region already disfavoured by LHC.

Moreover, we have obtained an analytical expansion of the dominant gluino amplitude by using a theorem of matrix algebra [59] and have arrived at the approximate master formula (3.9). This formula worked as a guide in order to understand better the cancellations between various contributions, decoupling effects and enhancement scenarios in $t \rightarrow q h$ amplitude. We conclude that the main enhancement for $\mathcal{B}(t \rightarrow c h)$ arises basically from the largeness of the parameters: $\left|\delta_{L R}^{32}\right| \sim\left|A_{U}^{32}\right| / M_{S}$ (and/or $\left.\left|\delta_{R L}^{32}\right|\right)$ and $\left|\delta_{R R}^{32}\right|$.

Numerical results depicted in figures 3 and 4 show that for $\left|A_{U}^{32}\right| / M_{S} \gtrsim O(1)$ or $\left|\delta_{R R}^{32}\right| \sim$ $O(1)$, the branching ratio $\mathcal{B}(t \rightarrow c h) \approx 10^{-5}$ is enhanced almost by 9 orders of magnitude w.r.t. SM expectation, but unfortunately it is still below the near future LHC sensitivity of (1.4). This is because of cancellations between leading order penguin and self energy diagrams, so that decoupling always takes place. Only in case where $\left|A_{U}^{32}\right| \gtrsim 8 M_{S}$ the branching ratio is approaching the expected LHC sensitivity. In such a case however CCB minima are likely to appear as we briefly showed in eq. (5.7) or (5.8).

For $t \rightarrow u h$ on the other hand, although in principle the decay rate is expected to be of the same order as with $t \rightarrow c h$, the neutron EDM constraints, induced from the CKM phase, severely suppress the allowed parameter space into a tuned region in which decay rates are small, $\mathcal{B}(t \rightarrow u h)<10^{-7}$, again far below experimental sensitivity.

We therefore conclude that an MSSM driven $\mathcal{B}(t \rightarrow q h)$ is unlikely to be observed even at high luminosity LHC. Apart from rather unnatural corners of the parameter space, the typical MSSM prediction, even for flavour changing insertions in the up sector of 
$\delta_{L R, R R} \sim \mathcal{O}(1)$, is $\mathcal{B}(t \rightarrow q h) \approx 10^{-8}-10^{-9}$. Although small, this is still five to six orders of magnitude above the SM expectation. If LHC discovers up-squarks and gluinos it will be vital to develop techniques that will take us to such small branching ratios for $t \rightarrow q h$ decay. If however LHC observes the rare $t \rightarrow q h$ decays at projected maximal sensitivity of about $10^{-4}$, their origin must probably lie in physics other than, or beyond, MSSM with R-parity conservation.

\section{Acknowledgments}

AD would like to thank Francesca Borzumati, Sven Heinemeyer and Howie Haber for useful discussions during SUSY-2014 conference. We would like to thank Wolfgang Altmannshofer for bringing to our attention refs. [73, 75, 79] for CCB constraints on flavour changing mass insertions. This research has been co-financed by the European Union (European Social Fund - ESF) and Greek national funds through the Operational Program "Education and Lifelong Learning" of the National Strategic Reference Framework (NSRF) - Research Funding Program: THALIS-Investing in the society of knowledge through the European Social Fund. J.R. would like to thank University of Ioannina and CERN for the hospitality during his stays there. His work was supported in part by the Polish National Science Center under the research grants DEC-2011/01/M/ST2/02466 and DEC2012/05/B/ST2/02597.

\section{A Explicit expressions for MSSM vertices}

Throughout the paper we follow the notation and conventions of refs. $[49,50]$, where the definitions of the Lagrangian parameters, mass matrices and mixing matrices used for their diagonalization are given for all MSSM sectors. Here for completeness we repeat just the explicit expressions for the couplings needed to calculate the effective $t \rightarrow q h$ vertex in section 2. For more details the reader is referred to more up-to-date ref. [50].

The CP-even Higgs boson mass rotation matrix $Z_{R}$ is defined in terms of commonly used angle- $\alpha$ as (also as usual $\tan \beta=\frac{v_{2}}{v_{1}}$ )

$$
Z_{R}=\left(\begin{array}{cc}
\cos \alpha & -\sin \alpha \\
\sin \alpha & \cos \alpha
\end{array}\right) .
$$

Matrices used to mass matrices of supersymmetric particles are defined, respectively, as:

$$
\begin{aligned}
Z_{-}^{T} M_{C} Z_{+} & =\operatorname{diag}\left(m_{\chi_{1}}, m_{\chi_{2}}\right) & & \text { chargino, } \\
Z_{N}^{T} M_{N} Z_{N} & =\operatorname{diag}\left(m_{\chi_{1}^{0}}, \ldots, m_{\chi_{4}^{0}}\right) & & \text { neutralino, } \\
Z_{D}^{\dagger} M_{D}^{2} Z_{D} & =\operatorname{diag}\left(m_{D_{1}}^{2}, \ldots, m_{D_{6}}^{2}\right) & & \text { down - squarks, } \\
Z_{U}^{T} M_{U}^{2} Z_{U}^{*} & =\operatorname{diag}\left(m_{U_{1}}^{2}, \ldots, m_{U_{6}}^{2}\right) & & \text { up - squarks, }
\end{aligned}
$$

where the expressions for $M_{C}, M_{N}, M_{D}^{2}, M_{U}^{2}$ can be found in ref. [50].

With the above definitions, relevant tree-level vertices can be written down as (summation from 1 to 3 over all repeating flavour indices $A, B, \ldots$ is always assumed): 
- Neutral CP-even Higgs-up quark coupling is:

$$
V_{u H u}^{I K I}=-\frac{1}{\sqrt{2}} Y_{u}^{I} Z_{R}^{2 K}
$$

where up-quark Yukawa coupling is $Y_{u}^{I}=\frac{\sqrt{2} m_{u}^{I}}{v_{2}}$.

- Couplings relevant for diagram with gluino exchange:

$$
\begin{aligned}
V_{H U U}^{K l i}= & -\frac{e^{2}}{3 c_{W}^{2}}\left(v_{1} Z_{R}^{1 K}-v_{2} Z_{R}^{2 K}\right)\left(\hat{I}^{l i}+\frac{3-8 s_{W}^{2}}{4 s_{W}^{2}} Z_{U}^{A l *} Z_{U}^{A i}\right) \\
& -v_{2}\left(Y_{u}^{A}\right)^{2} Z_{R}^{2 K}\left(Z_{U}^{A l *} Z_{U}^{A i}+Z_{U}^{(A+3) l *} Z_{U}^{(A+3) i}\right) \\
& +\frac{1}{\sqrt{2}} Z_{R}^{2 K}\left(A_{u}^{A B *} Z_{U}^{A l *} Z_{U}^{(B+3) i}+A_{u}^{A B} Z_{U}^{A i} Z_{U}^{(B+3) l *}\right) \\
& +\frac{1}{\sqrt{2}} Z_{R}^{1 K}\left(A_{u}^{\prime A B *} Z_{U}^{A l *} Z_{U}^{(B+3) i}+A_{u}^{A B} Z_{U}^{A i} Z_{U}^{(B+3) l *}\right) \\
& +\frac{1}{\sqrt{2}} Y_{u}^{A} Z_{R}^{1 K}\left(\mu^{*} Z_{U}^{A i} Z_{U}^{(A+3) l *}+\mu Z_{U}^{A l *} Z_{U}^{(A+3) i}\right), \\
V_{u U \tilde{g}, L}^{J j i}= & g_{3} \sqrt{2} T_{a b}^{i}\left(-Z_{U}^{J j *}\right) \\
V_{u U \tilde{g}, R}^{I j i}= & g_{3} \sqrt{2} T_{a b}^{i}\left(Z_{U}^{(I+3) j *}\right)
\end{aligned}
$$

where the generators $T^{i}$ are the Gell-Mann of SU(3) with Casimir invariant normalised to $C^{2}=\sum_{j} T^{j} T^{j}=\frac{4}{3} \hat{\mathbf{I}}$.

- Additional couplings necessary for neutralino mediated diagrams are:

$$
\begin{aligned}
V_{\chi^{0} H \chi^{0}, L}^{l K i}= & V_{\chi^{0} H \chi^{0}, R}^{i K l *}=\frac{e}{2 s_{W} c_{W}}\left(\left(Z_{R}^{1 K} Z_{N}^{3 i}-Z_{R}^{2 K} Z_{N}^{4 i}\right)\left(Z_{N}^{1 l} s_{W}-Z_{N}^{2 l} c_{W}\right)\right. \\
& \left.+\left(Z_{R}^{1 K} Z_{N}^{3 l}-Z_{R}^{2 k} Z_{N}^{4 l}\right)\left(Z_{N}^{1 i} s_{W}-Z_{N}^{2 i} c_{W}\right)\right), \\
V_{u U \chi^{0}, L}^{J j i}= & \frac{-e}{\sqrt{2} s_{W} c_{W}} Z_{U}^{I j \star}\left(\frac{1}{3} Z_{N}^{1 i} s_{W}+Z_{N}^{2 i} c_{W}\right)-Y_{u}^{I} Z_{U}^{(I+3) j \star} Z_{N}^{4 i} \\
V_{u U \chi^{0}, R}^{I j i}= & \frac{2 \sqrt{2} e}{3 c_{W}} Z_{U}^{(I+3) j \star} Z_{N}^{1 i \star}-Y_{u}^{I} Z_{U}^{I j \star} Z_{N}^{4 i \star} .
\end{aligned}
$$

- Couplings relevant for chargino mediated diagrams are:

$$
\begin{aligned}
V_{\chi H \chi, L}^{l K i}= & V_{\chi H \chi, R}^{i K l *}=-\frac{e}{\sqrt{2} s_{W}}\left(Z_{R}^{1 K} Z_{-}^{2 i} Z_{+}^{1 l}+Z_{R}^{2 K} Z_{-}^{1 i} Z_{+}^{2 l}\right), \\
V_{u D \chi, L}^{J j i}= & -\left(\frac{e}{s_{W}} Z_{D}^{A j} Z_{-}^{1 i}+Y_{d}^{A} Z_{D}^{A+3 j} Z_{-}^{2 i}\right) K^{J A *}, \\
V_{u D \chi, R}^{I j i}= & Y_{u}^{I} Z_{D}^{A j} Z_{+}^{2 i *} K^{I A *}, \\
V_{H D D}^{K l i}= & \frac{e^{2}}{6 c_{W}^{2}}\left(v_{1} Z_{R}^{1 K}-v_{2} Z_{R}^{2 K}\right)\left(\hat{I}^{l i}+\frac{3-4 s_{W}^{2}}{2 s_{W}^{2}} Z_{D}^{A l *} Z_{D}^{A i}\right) \\
& -v_{1}\left(Y_{d}^{A}\right)^{2} Z_{R}^{1 K}\left(Z_{D}^{A l *} Z_{D}^{A i}+Z_{D}^{(A+3) l *} Z_{D}^{(A+3) i}\right)
\end{aligned}
$$




\begin{tabular}{|c|c|}
\hline SLHA2 [82] & Ref. [49,50] \\
\hline$\hat{T}_{U}, \hat{T}_{D}, \hat{T}_{E}$ & $-A_{u}^{T},+A_{d}^{T},+A_{l}^{T}$ \\
$\hat{m}_{\tilde{Q}}^{2}, \hat{m}_{\tilde{L}}^{2}$ & $m_{Q}^{2}, m_{L}^{2}$ \\
$\hat{m}_{\tilde{u}}^{2}, \hat{m}_{\tilde{d}}^{2}, \hat{m}_{\tilde{l}}^{2}$ & $\left(m_{U}^{2}\right)^{T},\left(m_{D}^{2}\right)^{T},\left(m_{E}^{2}\right)^{T}$ \\
$\mathcal{M}_{\tilde{u}}^{2}, \mathcal{M}_{\tilde{d}}^{2}$ & $\left(\mathcal{M}_{U}^{2}\right)^{T},\left(\mathcal{M}_{D}^{2}\right)^{T}$ \\
\hline
\end{tabular}

Table 2. Comparison of SLHA2 [82] and refs. [49, 50] conventions.

$$
\begin{aligned}
& -\frac{1}{\sqrt{2}} Z_{R}^{1 K}\left(A_{d}^{A B *} Z_{D}^{A i} Z_{D}^{(B+3) l *}+A_{d}^{A B} Z_{D}^{A l *} Z_{D}^{(B+3) i}\right) \\
& +\frac{1}{\sqrt{2}} Z_{R}^{2 K}\left(A_{d}^{\prime A B *} Z_{D}^{A i} Z_{D}^{(B+3) l *}+A_{d}^{\prime A B} Z_{D}^{A l *} Z_{D}^{(B+3) i}\right) \\
& -\frac{1}{\sqrt{2}} Y_{d}^{A} Z_{R}^{2 K}\left(\mu^{*} Z_{D}^{A l *} Z_{D}^{(A+3) i}+\mu Z_{D}^{A i} Z_{D}^{(A+3) l *}\right),
\end{aligned}
$$

where $K$ is the Cabibbo-Kobayashi-Maskawa matrix and $Y_{d}^{I}=-\frac{\sqrt{2} m_{d}^{I}}{v_{1}}$.

One should also note that the conventions used in the paper, following refs. $[49,50]$ differ minimally from the now commonly accepted SLHA2 convention [82] for the MSSM parameters. However, translation of the soft breaking parameters (others do not differ at all) can be done immediately using information from table 2 .

\section{B Passarino-Veltman loop functions}

Our convention for Passarino-Veltman integral functions follows Axelrod's in ref. [83]. For the integrals entering directly our 1PI-irreducible amplitudes, we have the defining expressions for 2- and 3-point functions:

$$
\begin{aligned}
& \left\{B_{0}, B^{\mu}\right\}\left[k_{1}, m_{1}, m_{2}\right] \\
& \equiv \int \frac{d^{4} p}{(2 \pi)^{4}} \frac{\left\{1, p^{\mu}\right\}}{\left(p^{2}-m_{1}^{2}\right)\left(\left(p+k_{1}\right)^{2}-m_{2}^{2}\right)}, \\
& \left\{C_{0}, C^{\mu}, \tilde{C}_{0}\right\} \\
& {\left[k_{1}, k_{2}, m_{1}, m_{2}, m_{3}\right]} \\
& \equiv \int \frac{d^{4} p}{(2 \pi)^{4}} \frac{\left\{1, p^{\mu}, p^{2}\right\}}{\left(p^{2}-m_{1}^{2}\right)\left(\left(p+k_{1}\right)^{2}-m_{2}^{2}\right)\left(\left(p+k_{1}+k_{2}\right)^{2}-m_{3}^{2}\right)} .
\end{aligned}
$$

The expression above can be generalised to the case of general $n$-point 1-loop functions as:

$$
\begin{aligned}
& P V_{n}^{\mu_{1} \ldots \mu_{s}}\left[k_{1}, \ldots, k_{n-1}, m_{1}, \ldots, m_{n}\right]= \\
& \qquad \frac{d^{4} p}{(2 \pi)^{4}} \frac{p^{\mu_{1}} \ldots p^{\mu_{s}}}{\left(p^{2}-m_{1}^{2}\right) \prod_{j=2}^{n}\left(\left(p+k_{1}+\cdots+k_{j-1}\right)^{2}-m_{j}^{2}\right)}, \quad(n \geq 2) .
\end{aligned}
$$


Obviously, for $n=2,3$ one obtains the analytic expression for the $B, C$-functions given in eqs. (B.1) and (B.2) (with $\tilde{C}_{0}=g_{\mu \nu} P V_{3}^{\mu \nu}$ ). In standard notation higher order $n=4,5 \ldots$ functions are commonly denoted as $D, E, \ldots$-functions. Such higher order integrals are absent from the calculation of $t \rightarrow q h$ decays at one-loop [eqs. (2.10) and (2.13)], but they unavoidably arise in the flavour expansion approximation of eq. (3.7).

In practical calculations, it is usually more convenient to replace the tensorial integral functions by functions transforming as scalars under the Lorentz group. For the lowest vectorial functions they are defined through the relation

$$
\begin{array}{rlrl}
B^{\mu} & =k_{1}^{\mu} B_{1}, & & {\left[k_{1}, m_{1}, m_{2}\right]} \\
C^{\mu} & =k_{1}^{\mu} C_{11}+k_{2}^{\mu} C_{12}, & & {\left[k_{1}, k_{2}, m_{1}, m_{2}, m_{3}\right]} \\
\ldots & & \\
P V_{n}^{\mu} & =k_{1}^{\mu} \overline{P V}_{n}^{1}+\cdots+k_{n-1}^{\mu} \overline{P V}_{n}^{n-1}=\sum_{i=1}^{n-1} k_{i}^{\mu} \overline{P V}_{n}^{i}, & & {\left[k_{1} \ldots k_{n-1} ; m_{1} \ldots m_{n}\right]}
\end{array}
$$

and similarly for higher tensor functions. In our notation, all arguments, common for $\mathrm{PV}$-functions of equal order $n$, are displayed separately within the respective brackets.

For FC processes, where partial cancellations between topologically distinct diagrams take place, it is considerably more convenient to work in a different description of the PV-functions in which all arguments become dimensionless. As follows directly from the definition (B.3), PV loop integrals are homogeneous functions of their arguments:

$$
P V_{n}^{\mu_{1} \ldots \mu_{s}}\left[k_{1}, \ldots, k_{n-1}, m_{1}, \ldots, m_{n}\right]=M^{4+s-2 n} P V_{n}^{\mu_{1} \ldots \mu_{s}}\left[\frac{k_{1}}{M}, \ldots, \frac{k_{n-1}}{M}, \frac{m_{1}}{M}, \ldots, \frac{m_{n}}{M}\right]
$$

with $M$ being an arbitrary mass scale, usually chosen as a typical scale for a given loop diagram.

A useful property associates differences of integral functions of a certain order with integral functions of next order. For example, as can be directly verified from the definitions in eqs. (B.1) and (B.2), one has

$$
\frac{B_{0}\left[k_{1}, m_{1}, M_{2}\right]-B_{0}\left[k_{1}, m_{1}^{\prime}, M_{2}\right]}{m_{1}^{2}-m_{1}^{\prime 2}}=C_{0}\left[0, k_{1}, m_{1}, m_{1}^{\prime}, M_{2}\right] .
$$

In general case this relation has the following structure:

$$
\begin{gathered}
\frac{P V_{n}^{X}\left[k_{1} \ldots k_{n-1} ; m_{1} \ldots M_{n}\right]-P V_{n}^{X}\left[k_{1} \ldots k_{n-1} ; m_{1}^{\prime} \ldots M_{n}\right]}{m_{1}^{2}-m_{1}^{\prime 2}} \\
\quad=P V_{n+1}^{X}\left[0, k_{1} \ldots k_{n-1} ; m_{1}, m_{1}^{\prime} \ldots M_{n}\right], \\
\frac{P V_{n}^{X}\left[\ldots k_{j-1} \ldots ; \ldots m_{j} \ldots\right]-P V_{n}^{X}\left[\ldots k_{j-1} \ldots ; \ldots m_{j}^{\prime} \ldots\right]}{m_{j}^{2}-m_{j}^{\prime 2}} \\
=P V_{n+1}^{X}\left[\ldots k_{j-1}, 0 \ldots ; \ldots m_{j}, m_{j}^{\prime} \ldots\right],(j \geq 2),
\end{gathered}
$$

with $X$ being any set of Lorentz indices of momenta in the numerator of loop integrand. 
For auxiliary scalar functions, defined in eqs. (B.4)-(B.6) this property manifests in a slightly more complicated manner. That is, depending on the position of $m_{j}, m_{j}^{\prime}$ within the brackets, the differences of $\overline{P V}_{n}^{i}$ can either produce $\overline{P V}_{n+1}^{i}$ or $\overline{P V}_{n+1}^{i+1}$. For the lowest order integrals, this property has the suggestive form

$$
\begin{aligned}
& \frac{B_{1}\left[k_{1}, m_{1}, M_{2}\right]-B_{1}\left[k_{1}, m_{1}^{\prime}, M_{2}\right]}{m_{1}^{2}-m_{1}^{\prime 2}}=C_{12}\left[0, k_{1}, m_{1}, m_{1}^{\prime}, M_{2}\right], \\
& \frac{B_{1}\left[k_{1}, M_{1}, m_{2}\right]-B_{1}\left[k_{1}, M_{1}, m_{2}^{\prime}\right]}{m_{2}^{2}-m_{2}^{\prime 2}}=C_{11}\left[k_{1}, 0, M_{1}, m_{2}, m_{2}^{\prime}\right] .
\end{aligned}
$$

For any order of scalar PV functions defined in (B.6) one has

$$
\begin{aligned}
\frac{\overline{P V}_{n}^{i}\left[\ldots k_{j-1} \ldots ; \ldots m_{j} \ldots\right]-\overline{P V}_{n}^{i}\left[\ldots k_{j-1} \ldots ; \ldots m_{j}^{\prime} \ldots\right]}{m_{j}^{2}-m_{j}^{\prime 2}} & \\
\stackrel{(j>i)}{=} \overline{P V}_{n+1}^{i}\left[\ldots k_{j-1}, 0 \ldots ; \ldots m_{j}, m_{j}^{\prime} \ldots\right] & \stackrel{(j \leq i)}{=} \overline{P V}_{n+1}^{i+1}\left[\ldots k_{j-1}, 0 \ldots ; \ldots m_{j}, m_{j}^{\prime} \ldots\right] .
\end{aligned}
$$

Formulae (B.7)-(B.12) are particularly useful because their r.h.s. 's are explicitly regular in the limit of degenerate masses (as all functions defined by 1-loop integrals). Thus, they allow to generalise (3.6) to the case of mass matrices with degenerated diagonal elements.

Open Access. This article is distributed under the terms of the Creative Commons Attribution License (CC-BY 4.0), which permits any use, distribution and reproduction in any medium, provided the original author(s) and source are credited.

\section{References}

[1] DONUT collaboration, K. Kodama et al., Observation of $\tau$ neutrino interactions, Phys. Lett. B 504 (2001) 218 [hep-ex/0012035] [INSPIRE].

[2] D0 collaboration, S. Abachi et al., Observation of the top quark, Phys. Rev. Lett. 74 (1995) 2632 [hep-ex/9503003] [INSPIRE].

[3] CDF collaboration, F. Abe et al., Observation of top quark production in $\bar{p} p$ collisions, Phys. Rev. Lett. 74 (1995) 2626 [hep-ex/9503002] [INSPIRE].

[4] F. Englert and R. Brout, Broken symmetry and the mass of gauge vector mesons, Phys. Rev. Lett. 13 (1964) 321 [INSPIRE].

[5] P.W. Higgs, Broken symmetries and the masses of gauge bosons, Phys. Rev. Lett. 13 (1964) 508 [INSPIRE].

[6] G.S. Guralnik, C.R. Hagen and T.W.B. Kibble, Global conservation laws and massless particles, Phys. Rev. Lett. 13 (1964) 585 [INSPIRE].

[7] CMS collaboration, Observation of a new boson at a mass of $125 \mathrm{GeV}$ with the CMS experiment at the LHC, Phys. Lett. B 716 (2012) 30 [arXiv:1207.7235] [INSPIRE]. 
[8] ATLAS collaboration, Observation of a new particle in the search for the standard model Higgs boson with the ATLAS detector at the LHC, Phys. Lett. B 716 (2012) 1 [arXiv:1207.7214] [INSPIRE].

[9] S. Weinberg, A model of leptons, Phys. Rev. Lett. 19 (1967) 1264 [inSPIRE].

[10] G. Eilam, J.L. Hewett and A. Soni, Rare decays of the top quark in the standard and two Higgs doublet models, Phys. Rev. D 44 (1991) 1473 [Erratum ibid. D 59 (1999) 039901] [INSPIRE].

[11] B. Mele, S. Petrarca and A. Soddu, A new evaluation of the $t \rightarrow c H$ decay width in the standard model, Phys. Lett. B 435 (1998) 401 [hep-ph/9805498] [InSPIRE].

[12] S.L. Glashow, J. Iliopoulos and L. Maiani, Weak interactions with lepton-hadron symmetry, Phys. Rev. D 2 (1970) 1285 [inSPIRE].

[13] H.P. Nilles, Supersymmetry, supergravity and particle physics, Phys. Rept. 110 (1984) 1 [INSPIRE].

[14] H.E. Haber and G.L. Kane, The search for supersymmetry: probing physics beyond the standard model, Phys. Rept. 117 (1985) 75 [INSPIRE].

[15] S.P. Martin, A supersymmetry primer, Adv. Ser. Direct. High Energy Phys. 21 (2010) 1 [hep-ph/9709356] [INSPIRE].

[16] J. Guasch and J. Solà, FCNC top quark decays: a door to SUSY physics in high luminosity colliders?, Nucl. Phys. B 562 (1999) 3 [hep-ph/9906268] [INSPIRE].

[17] J.J. Cao, G. Eilam, M. Frank, K. Hikasa, G.L. Liu et al., SUSY-induced FCNC top-quark processes at the large hadron collider, Phys. Rev. D 75 (2007) 075021 [hep-ph/0702264] [INSPIRE].

[18] J. Cao, G. Eilam, K.-i. Hikasa and J.M. Yang, Experimental constraints on stop-scharm flavor mixing and implications in top-quark FCNC processes, Phys. Rev. D 74 (2006) 031701 [hep-ph/0604163] [INSPIRE].

[19] J.L. Diaz-Cruz, H.-J. He and C.P. Yuan, Soft SUSY breaking, stop scharm mixing and Higgs signatures, Phys. Lett. B 530 (2002) 179 [hep-ph/0103178] [INSPIRE].

[20] J. Cao, C. Han, L. Wu, J.M. Yang and M. Zhang, SUSY induced top quark FCNC decay $t \rightarrow$ ch after Run I of LHC, Eur. Phys. J. C 74 (2014) 3058 [arXiv:1404.1241] [inSPIRE].

[21] ATLAS collaboration, Search for top quark decays $t \rightarrow q H$ with $H \rightarrow \gamma \gamma$ using the ATLAS detector, JHEP 06 (2014) 008 [arXiv:1403.6293] [INSPIRE].

[22] CMS Collaboration, Combined multilepton and diphoton limit on $t$ to $\mathrm{cH}$, CMS-PAS-HIG-13-034 (2013).

[23] Top Quark Working Group collaboration, K. Agashe et al., Working Group Report: Top Quark, arXiv:1311.2028 [INSPIRE].

[24] ATLAS collaboration, Measurements of the properties of the Higgs-like boson in the two photon decay channel with the ATLAS detector using $25 \mathrm{fb}^{-1}$ of proton-proton collision data, ATLAS-CONF-2013-012 (2013).

[25] J.A. Aguilar-Saavedra and G.C. Branco, Probing top flavor changing neutral scalar couplings at the CERN LHC, Phys. Lett. B 495 (2000) 347 [hep-ph/0004190] [INSPIRE].

[26] J.A. Aguilar-Saavedra, Top flavor-changing neutral interactions: Theoretical expectations and experimental detection, Acta Phys. Polon. B 35 (2004) 2695 [hep-ph/0409342] [INSPIRE]. 
[27] C. Kao, H.-Y. Cheng, W.-S. Hou and J. Sayre, Top decays with flavor changing neutral Higgs interactions at the LHC, Phys. Lett. B 716 (2012) 225 [arXiv:1112.1707] [INSPIRE].

[28] Y. Wang, F.P. Huang, C.S. Li, B.H. Li, D.Y. Shao et al., Constraints on flavor-changing neutral-current Htq couplings from the signal of $t H$ associated production with $Q C D$ next-to-leading order accuracy at the LHC, Phys. Rev. D 86 (2012) 094014 [arXiv: 1208.2902] [INSPIRE].

[29] N. Craig et al., Searching for $t \rightarrow$ ch with multi-leptons, Phys. Rev. D 86 (2012) 075002 [arXiv: 1207.6794] [INSPIRE].

[30] K.-F. Chen, W.-S. Hou, C. Kao and M. Kohda, When the Higgs meets the top: search for $t \rightarrow c h^{0}$ at the LHC, Phys. Lett. B 725 (2013) 378 [arXiv: 1304.8037] [INSPIRE].

[31] D. Atwood, S.K. Gupta and A. Soni, Constraining the flavor changing Higgs couplings to the top-quark at the LHC, JHEP 1410 (2014) 57 [arXiv:1305.2427] [INSPIRE].

[32] M. Gorbahn and U. Haisch, Searching for $t \rightarrow c(u) h$ with dipole moments, JHEP 06 (2014) 033 [arXiv: 1404.4873] [INSPIRE].

[33] A. Greljo, J.F. Kamenik and J. Kopp, Disentangling flavor violation in the top-Higgs sector at the LHC, JHEP 07 (2014) 046 [arXiv: 1404.1278] [INSPIRE].

[34] L. Wu, Enhancing thj production from top-Higgs FCNC couplings, arXiv:1407.6113 [INSPIRE].

[35] L. Girardello and M.T. Grisaru, Soft breaking of supersymmetry, Nucl. Phys. B 194 (1982) 65 [INSPIRE].

[36] L.J. Hall and L. Randall, Weak scale effective supersymmetry, Phys. Rev. Lett. 65 (1990) 2939 [INSPIRE].

[37] F. Borzumati, G.R. Farrar, N. Polonsky and S.D. Thomas, Soft Yukawa couplings in supersymmetric theories, Nucl. Phys. B 555 (1999) 53 [hep-ph/9902443] [INSPIRE].

[38] J.P.J. Hetherington, The spectrum of the MSSM with nonstandard supersymmetry breaking, JHEP 10 (2001) 024 [hep-ph/0108206] [INSPIRE].

[39] G. D'Ambrosio, G.F. Giudice, G. Isidori and A. Strumia, Minimal flavor violation: an effective field theory approach, Nucl. Phys. B 645 (2002) 155 [hep-ph/0207036] [INSPIRE].

[40] A. Dery, A. Efrati, Y. Nir, Y. Soreq and V. Susič, Model building for flavor changing Higgs couplings, arXiv:1408.1371 [INSPIRE].

[41] F. Gabbiani, E. Gabrielli, A. Masiero and L. Silvestrini, A complete analysis of FCNC and CP constraints in general SUSY extensions of the standard model, Nucl. Phys. B 477 (1996) 321 [hep-ph/9604387] [inSPIRE].

[42] M. Misiak, S. Pokorski and J. Rosiek, Supersymmetry and FCNC effects, Adv. Ser. Direct. High Energy Phys. 15 (1998) 795 [hep-ph/9703442] [INSPIRE].

[43] G.F. Giudice, M. Nardecchia and A. Romanino, Hierarchical soft terms and flavor physics, Nucl. Phys. B 813 (2009) 156 [arXiv:0812.3610] [INSPIRE].

[44] J. Rosiek, P. Chankowski, A. Dedes, S. Jager and P. Tanedo, SUSY_FLAVOR: a computational tool for FCNC and CP-violating processes in the MSSM, Comput. Phys. Commun. 181 (2010) 2180 [arXiv: 1003.4260] [INSPIRE]. 
[45] A. Crivellin et al., SUSY_FLAVOR v2: a computational tool for FCNC and CP-violating processes in the MSSM, Comput. Phys. Commun. 184 (2013) 1004 [arXiv:1203.5023] [INSPIRE].

[46] J. Rosiek, SUSY FLAVOR v2.5: a computational tool for FCNC and CP-violating processes in the MSSM, arXiv:1410.0606 [INSPIRE].

[47] C. Zhang and F. Maltoni, Top-quark decay into Higgs boson and a light quark at next-to-leading order in QCD, Phys. Rev. D 88 (2013) 054005 [arXiv:1305.7386] [INSPIRE].

[48] A. Czarnecki, J.G. Korner and J.H. Piclum, Helicity fractions of $W$ bosons from top quark decays at NNLO in QCD, Phys. Rev. D 81 (2010) 111503 [arXiv:1005.2625] [InSPIRE].

[49] J. Rosiek, Complete set of Feynman rules for the minimal supersymmetric extension of the standard model, Phys. Rev. D 41 (1990) 3464 [InSPIRE].

[50] J. Rosiek, Complete set of Feynman rules for the MSSM: erratum, hep-ph/9511250 [INSPIRE].

[51] A. Dedes, J. Rosiek and P. Tanedo, Complete one-loop MSSM predictions for B $\rightarrow$ lepton lepton' at the Tevatron and LHC, Phys. Rev. D 79 (2009) 055006 [arXiv:0812.4320] [INSPIRE].

[52] A. Crivellin, Effective Higgs vertices in the generic MSSM, Phys. Rev. D 83 (2011) 056001 [arXiv: 1012.4840] [INSPIRE].

[53] A. Crivellin, L. Hofer and J. Rosiek, Complete resummation of chirally-enhanced loop-effects in the MSSM with non-minimal sources of flavor-violation, JHEP 07 (2011) 017 [arXiv:1103.4272] [INSPIRE].

[54] J.F. Gunion, H.E. Haber, G.L. Kane and S. Dawson, The Higgs hunter's guide, Front. Phys. 80 (2000) 1 [INSPIRE].

[55] A. Djouadi, Implications of the Higgs discovery for the MSSM, Eur. Phys. J. C 74 (2014) 2704 [arXiv: 1311.0720] [INSPIRE].

[56] ATLAS collaboration, Search for charged Higgs bosons in the $\tau+$ jets final state with $p p$ collision data recorded at $\sqrt{s}=8 \mathrm{TeV}$ with the ATLAS experiment, ATLAS-CONF-2013-090 (2013).

[57] M. Drees, A supersymmetric explanation of the excess of Higgs-like events at the LHC and at LEP, Phys. Rev. D 86 (2012) 115018 [arXiv:1210.6507] [INSPIRE].

[58] A.J. Buras, A. Romanino and L. Silvestrini, $K \rightarrow$ pi neutrino anti-neutrino: a model independent analysis and supersymmetry, Nucl. Phys. B 520 (1998) 3 [hep-ph/9712398] [INSPIRE].

[59] A. Dedes, M. Paraskevas, J. Rosiek, K. Suxho and K. Tamvakis, Mass insertion vs. mass eigenstate calculations in flavour physics, work in progress.

[60] Particle Data Group collaboration, J. Beringer et al., Review of particle physics, Phys. Rev. D 86 (2012) 010001 [INSPIRE].

[61] ATLAS collaboration, Search for new phenomena in final states with large jet multiplicities and missing transverse momentum at $\sqrt{s}=8 \mathrm{TeV}$ proton-proton collisions using the ATLAS experiment, JHEP 10 (2013) 130 [Erratum ibid. 1401 (2014) 109] [arXiv:1308.1841] [INSPIRE]. 
[62] A. Delgado, G.F. Giudice, G. Isidori, M. Pierini and A. Strumia, The light stop window, Eur. Phys. J. C 73 (2013) 2370 [arXiv: 1212.6847] [InSPIRE].

[63] M.R. Buckley, T. Plehn and M.J. Ramsey-Musolf, Stop on top, Phys. Rev. D 90 (2014) 014046 [arXiv: 1403.2726] [INSPIRE].

[64] C.A. Baker et al., An improved experimental limit on the electric dipole moment of the neutron, Phys. Rev. Lett. 97 (2006) 131801 [hep-ex/0602020] [INSPIRE].

[65] S. Heinemeyer, W. Hollik and G. Weiglein, The mass of the lightest MSSM Higgs boson: a compact analytical expression at the two loop level, Phys. Lett. B 455 (1999) 179 [hep-ph/9903404] [INSPIRE].

[66] H.E. Haber, R. Hempfling and A.H. Hoang, Approximating the radiatively corrected Higgs mass in the minimal supersymmetric model, Z. Phys. C 75 (1997) 539 [hep-ph/9609331] [INSPIRE].

[67] J.M. Frere, D.R.T. Jones and S. Raby, Fermion masses and induction of the weak scale by supergravity, Nucl. Phys. B 222 (1983) 11 [INSPIRE].

[68] C. Kounnas, A.B. Lahanas, D.V. Nanopoulos and M. Quirós, Low-energy behavior of realistic locally supersymmetric grand unified theories, Nucl. Phys. B 236 (1984) 438 [InSPIRE].

[69] J.F. Gunion, H.E. Haber and M. Sher, Charge/color breaking minima and a-parameter bounds in supersymmetric models, Nucl. Phys. B 306 (1988) 1 [INSPIRE].

[70] J.A. Casas, A. Lleyda and C. Muñoz, Strong constraints on the parameter space of the MSSM from charge and color breaking minima, Nucl. Phys. B 471 (1996) 3 [hep-ph/9507294] [INSPIRE].

[71] A. Riotto and E. Roulet, Vacuum decay along supersymmetric flat directions, Phys. Lett. B 377 (1996) 60 [hep-ph/9512401] [INSPIRE].

[72] A. Kusenko, P. Langacker and G. Segre, Phase transitions and vacuum tunneling into charge and color breaking minima in the MSSM, Phys. Rev. D 54 (1996) 5824 [hep-ph/9602414] [INSPIRE].

[73] J.A. Casas and S. Dimopoulos, Stability bounds on flavor violating trilinear soft terms in the MSSM, Phys. Lett. B 387 (1996) 107 [hep-ph/9606237] [INSPIRE].

[74] C. Le Mouel, Optimal charge and color breaking conditions in the MSSM, Nucl. Phys. B 607 (2001) 38 [hep-ph/0101351] [INSPIRE].

[75] J.-h. Park, Metastability bounds on flavour-violating trilinear soft terms in the MSSM, Phys. Rev. D 83 (2011) 055015 [arXiv: 1011.4939] [INSPIRE].

[76] J.E. Camargo-Molina, B. O'Leary, W. Porod and F. Staub, Stability of the CMSSM against sfermion VEVs, JHEP 12 (2013) 103 [arXiv:1309.7212] [INSPIRE].

[77] N. Blinov and D.E. Morrissey, Vacuum stability and the MSSM Higgs mass, JHEP 03 (2014) 106 [arXiv: 1310.4174] [INSPIRE].

[78] D. Chowdhury, R.M. Godbole, K.A. Mohan and S.K. Vempati, Charge and color breaking constraints in MSSM after the Higgs discovery at LHC, JHEP 02 (2014) 110 [arXiv:1310.1932] [INSPIRE].

[79] W. Altmannshofer, C. Frugiuele and R. Harnik, Fermion hierarchy from sfermion anarchy, arXiv: 1409.2522 [INSPIRE]. 
[80] J.E. Camargo-Molina, B. O'Leary, W. Porod and F. Staub, Vevacious: a tool for finding the global minima of one-loop effective potentials with many scalars, Eur. Phys. J. C 73 (2013) 2588 [arXiv: 1307.1477] [INSPIRE].

[81] S. Pokorski, J. Rosiek and C.A. Savoy, Constraints on phases of supersymmetric flavor conserving couplings, Nucl. Phys. B 570 (2000) 81 [hep-ph/9906206] [INSPIRE].

[82] B.C. Allanach et al., SUSY Les Houches accord 2, Comput. Phys. Commun. 180 (2009) 8 [arXiv:0801.0045] [INSPIRE].

[83] A. Axelrod, Flavor changing Z0 decay and the top quark, Nucl. Phys. B 209 (1982) 349 [INSPIRE]. 